\title{
Surface photometry of emission-line galaxies in low density regions ${ }^{\star}$
}

\author{
J. Vennik ${ }^{1}$, U. Hopp ${ }^{2}$, and C.C. Popescu ${ }^{3,4}$ \\ 1 Tartu Observatory, 61602 Tõravere, Tartumaa, Estonia \\ e-mail: vennik@aai.ee \\ 2 Universitätssternwarte München, Scheiner Str. 1, D-81679 München, Germany \\ e-mail: hopp@usm.uni-muenchen.de \\ 3 Max Planck Institut für Kernphysik, Saupfercheckweg 1, D-69117 Heidelberg, Germany \\ e-mail: Cristina.Popescu@mpi-hd.mpg.de \\ 4 The Astronomical Institute of the Romanian Academy, Str. Cuţitul de Argint 5, 75212 Bucharest, Romania
}

Received November 12; accepted December 22, 1999

\begin{abstract}
Detailed $B$ and $R$ surface photometry is performed for 139 faint emission-line galaxies (ELGs) and for 2 non-ELGs. They are a subsample of the ELG sample selected by Popescu et al. $(1996,1998)$ towards four nearby voids. We derived the isophotal and integral photometric parameters and radial surface brightness (SB) profiles. Our sample consists mostly of low luminosity $\left(<M_{B}>_{\text {med }}=-17.7\right)$, small linear size $\left(<d_{25}>_{\text {med }}=\right.$ $6.0 \mathrm{kpc})\left(H_{0}=75 \mathrm{~km} \mathrm{~s}^{-1} \mathrm{Mpc}^{-1}\right)$ and blue $(<B-R\rangle_{\text {med }}$ $=0.93)$ galaxies. About $83 \%$ of the studied ELGs belong to the class of Blue Compact Galaxies (BCGs); others are luminous Starburst Nucleus Galaxies (SBNs) and a few interacting pairs. $72 \%$ of the ELGs possess a single dominant $\mathrm{H}$ II region, while the remaining $28 \%$ show multiple knots. Furthermore $71 \%$ of the ELGs show regular outer isophotes, and the remaining $29 \%$ reveal disturbed outer morphologies.
\end{abstract}

We performed the SB profile decomposition by means of disentangling the underlying stellar disk and the excess light above the exponential disk. We classified the profiles into 5 types: pure disk profiles (21\% of studied 139 ELGs); profiles that show both disk and bulge/nucleus/H II knot components (37\%); profiles that have central light depression (13\%); non-disky (concave) profiles (7\%); and almost unresolved (quasi-stellar) images and stellar SB profiles $(22 \%)$.

Popescu et al. (1997) found that most of the studied ELGs follow the spatial distribution of "normal" galaxies from the CfA Redshift Survey. 15 ELGs and 1 nonELG were classified as being well isolated and are distributed within nearby $(z \leq 0.033)$ voids. We found that these isolated ELGs are almost all dwarf galaxies with

\footnotetext{
Send offprint requests to: J. Vennik

* Based on observations obtained at the Calar Alto observatory, Almeria, Spain.
}

$-18.4<M_{B}<-12.7$, they show similar luminosities and colours as the nearby non-isolated ELGs. For 8 of 15 isolated ELGs an underlying LSBG has been detected. The underlying disks of isolated ELGs tend to be larger and of lower SB, than the disks of non-isolated ELGs (of the same total blue flux).

Key words: galaxies: photometry - galaxies: fundamental parameters - galaxies: structure; cosmology: large scale structure of Universe

\section{Introduction}

According to present knowledge, the large scale structure of the Universe, as derived from recent redshift surveys, reveals large inhomogenities and a complicated void-supercluster network. This picture of the large scale structure of the Universe is mainly shown by the distribution of bright galaxies. But whether the giant luminous galaxies are fair tracers of the large scale structure and whether the voids are really empty has been subject of intense debate. According to the biased galaxy formation scenarios (i.e. Dekel \& Silk 1986) the dwarf galaxies should trace the underlying dark matter and are expected to fill the voids.

A search for faint galaxies in voids was accomplished by Hopp et al. (1995) and Kuhn et al. (1997), with the intention to overcome the limitations of previous surveys in magnitude, diameter and surface brightness. As a result, a sample of galaxies of late morphological type and of low surface brightness was identified, with several very isolated galaxies and galaxies populating the rims of the voids. Vennik et al. (1996) performed $B$ and $R$ surface photometry of a subsample of these galaxies and concluded that 
both isolated and non-isolated galaxies show similar photometric and structural characteristics.

There are also interesting results from the study of the spatial distribution of emission-line galaxies (ELGs) (Salzer 1989; Weistrop et al. 1992, etc.). The ELGs are intrinsically small, very compact and with low luminosities. They look often almost stellar with no obvious underlying galaxy. Such galaxies can easily be missed by morphological surveys and, thus, are good candidates to fill up the voids. There have been even suggestions that a few ELGs have been found in the voids. Much effort has been concentrated on the study of the famous Bootes void, where several dozen of ELGs have been detected (Weistrop et al. 1995; Szomoru et al. 1996). The ELGs inside the Bootes void are nevertheless luminous galaxies, with strong star formation activity and not the faint objects predicted to be found in voids.

A new sample of ELGs was identified on the IIIa-J objective prism plates towards nearby voids by Popescu et al. $(1996,1997,1998)$. The objects were mainly selected by the presence of the high-excitation [O III] $\lambda 5007$ emission line. Redshifts were measured for all the ELGs and their spatial distribution and isolation was evaluated from the cone diagrams together with the calculated nearestneighbour distances (Popescu et al. 1997). A subsample of 16 isolated galaxies was found, with the isolated galaxies distributed within the nearby voids.

For most of the identified ELGs CCD photometry was obtained in the Johnson $B$ and Cousins $R$ bands. Here we present the surface photometry of a subsample of 139 ELGs (and 2 non-ELGs) and we discuss their photometric properties. A more detailed analyse of the new data and a study of the environment influence on the photometric properties of the ELGs will make the object of a separate paper. In Sect. 2 the observations and reductions are described. Section 3 deals with the surface photometry algorithm; Sect. 4 evaluates the integral consistency of our results. Section 5 outlines the morphological classification and profile fitting. Some "snap-shot statistics" are fulfilled in Sect. 6 and Sect. 7 summarizes the obtained results.

A Hubble constant of $H_{0}=75 \mathrm{~km} \mathrm{~s}^{-1} \mathrm{Mpc}^{-1}$ is used throughout of this paper.

\section{Observations and reduction of the frames}

\section{Observations}

The broad band $B$ and $R$ frames were taken during three observing runs in February 1995, May 1996 and June 1997 with the CAFOS focal reducer CCD camera attached to the Cassegrain focus of the Calar-Alto $2.2 \mathrm{~m}$ telescope. Some details of the observations are given in Table 1.

Most of the data were either obtained for aquisition purpose of the following spectroscopy or as a snapshot survey to obtain total magnitudes. Typical exposure time for every observing run is printed bold in Table 1 . Dedicated deeper (900 s $B$ and $600 \mathrm{~s} R$ ) images were observed for all isolated ELGs. A set of bias and flat frames for CCD corrections was taken, as usual. For magnitude calibrations and colour corrections we observed during each night a number of fields with standard stars in the star clusters M 92, NGC 2264, NGC 2419, NGC 4147 and NGC 7790 (Christian et al. 1985). During the May 1996 campaign the spectrophotometric standard stars HZ 21, HZ 44, and BD +33\#2642 were observed and used for photometric calibrations.

Zeropoint magnitudes $\left(c_{0, i}\right)$ and colour coefficients $\left(c_{1, i}\right)$ of the form

$B=b+c_{0, B}+c_{1, B}(b-r)-c_{2, B} X$,

$R=r+c_{0, R}+c_{1, R}(b-r)-c_{2, R} X$,

were fitted to the instrumental data of every observing night, where $B, R$ are standard magnitudes, $b, r$ are the instrumental magnitudes and $X$ is the airmass. We found a significant colour coefficient $c_{1, B}$ during all three observing runs and applied it to the magnitude calibration. We used the following mean values of the extinction coefficients: $c_{2, B}=0.2$ and $c_{2, R}=0.1$.

\section{Data reduction and filtering}

The raw CCD frames were de-biased and flat-fieldcorrected, as described by Stickel et al. (1993). Further data reduction was done by means of the Potsdam Image Processing System (PIPS) running within MIDAS environment. The bias and flat-field corrected CCD frames were searched for cosmic ray hits and possible "hot" and "cold" pixels by looking for pixel values above the expected noise and checking if they had a point spread function smaller than the estimated seeing. By means of Laplace filtering such faulty pixels were detected, masked out and replaced by the mean value of the surrounding area using the background interpolation routine of the PIPS. To improve the signal-to-noise ratio, we applied the adaptive filtering technique described in Lorenz et al. (1993). The main advantage of this technique consists in recognition of the local signal resolution and adapting its own impulse response to this resolution. The maximum filter size and the strength of the filter are variable. We varied the maximum filter size between $15 \times 15$ pixels and $23 \times 23$ pixels depending on the quality of the frames. The filter strength, defined by the minimum signal to noise ratio for the detection of a local signal, was generally between $2.0-2.5 \sigma$, where $\sigma$ is the rms noise level of the sky background at each scale length.

After the filtering, a careful sky background level determination and subtraction was performed on the smoothed frame, as described in more detail in Vennik et al. (1996). After the background subtraction the galaxy image was extracted from the large CCD frame 
Table 1. Dates and set-up of direct imaging with the Calar Alto $2.2 \mathrm{~m}$ telescope and CAFOS

\begin{tabular}{|c|c|c|c|c|c|c|c|c|}
\hline Dates & $\begin{array}{l}\text { Detector } \\
\text { type }\end{array}$ & $\begin{array}{l}\text { Detector } \\
\text { size }\end{array}$ & $\begin{array}{c}\text { Pixel } \\
\text { size } \\
{[\mu \mathrm{m}]} \\
\end{array}$ & $\begin{array}{l}\text { Pixel } \\
\text { scale } \\
{\left[{ }^{\prime \prime} / \mathrm{px}\right]} \\
\end{array}$ & $\begin{array}{c}\text { Photm. } \\
\text { band }\end{array}$ & $\begin{array}{l}\text { Exposure } \\
\text { time } \\
{[\mathrm{s}]} \\
\end{array}$ & $\begin{array}{c}\text { Sky } \\
\text { bright. } \\
{\left[\frac{\mathrm{mag}}{\square^{\prime \prime}}\right]}\end{array}$ & $\begin{array}{c}\text { Seeing } \\
\text { FWHM } \\
{\left[{ }^{\prime \prime}\right]}\end{array}$ \\
\hline 1995, Feb. 02-03 & Tek\#13 & $1024 \times 1024$ & 24 & 0.489 & $\begin{array}{l}\mathrm{B} \\
\mathrm{R}\end{array}$ & $\begin{array}{l}\mathbf{6 0}, 600 \\
\mathbf{6 0}\end{array}$ & $\begin{array}{l}22.4 \\
20.9\end{array}$ & $\begin{array}{l}1.8 \\
2.1\end{array}$ \\
\hline 1996, May 20-24 & Loral 8 & $1024 \times 1024$ & 15 & 0.333 & B & $\mathbf{6 0}, 600$ & 22.3 & 1.7 \\
\hline 1997, June $12-15$ & Site\#1d & $2048 \times 2048$ & 24 & 0.531 & $\begin{array}{l}\mathrm{B} \\
\mathrm{R}\end{array}$ & $\begin{array}{l}300, \mathbf{6 0 0}, 900 \\
150, \mathbf{3 0 0}, 600\end{array}$ & $\begin{array}{l}22.0 \\
20.9\end{array}$ & $\begin{array}{l}1.5 \\
1.5\end{array}$ \\
\hline
\end{tabular}

and interactively cleaned from disturbing objects like e.g. foreground stars projected onto the galaxy. We applied an interactive polygon editor for this cleaning procedure.

\section{Surface photometry and profile extraction}

The main results of the present study are the surface brightness (SB) profiles as well as a set of isophotal and integral parameters, derived from these profiles. The SB distribution was analyzed by two methods. First, we calculated the equivalent light profile by means of PIPS, by slicing the smoothed galaxy image at predefined intensity levels and counting the pixel intensities in areas between successive isophotes. The differential counts reduced to unit area give the azimuthally averaged intensity profile of the galaxy as a function of the equivalent radius, as defined in the RC2 (de Vaucouleurs et al. 1976). This procedure permits us to process the SB profile in faint outskirts of galaxies up to about $27.5 \mathrm{~B} \mathrm{mag} / \square^{\prime \prime}$ on our snap-shot $(60 \mathrm{~s})$ data frames. A set of isophotal, effective and asymptotic photometric parameters were determined on the basis of the equivalent light profiles and of the light growth curves.

The second approach for the surface photometry should recover the shape and orientation of isophotes. For this purpose we used the ellipse fitting algorithm of Bender \& Möllenhoff (1987) as available in the SURFPHOT package, running within MIDAS. The resulting fit was used to construct a smooth galaxy model. The subtraction of the elliptical model from the observed image allows us to detect underlying structures like spiral arms, dust, H II regions, if any.

As a result of the ellipse fitting we obtained a set of radial profiles: surface brightness (SB), minor-to-major axis ratio $(b / a)$, position angle $(\mathrm{PA})$ in each particular passband. The colour index profile was calculated by means of combining the $B$ and $R$ surface brightness profiles, which were obtained with one particular set of fitting ellipses applied both to the $B$ and $R$ frames. Four examples of the resulting profile-sets are shown in Fig. 1. They are se- lected in order to demonstrate the different profile types $\left(\right.$ see Sect. 5) ${ }^{1}$.

The observed photometric parameters, as measured from radial profiles, are given in Tables 4,5 . Table 4 summarizes the data of 125 galaxies, observed in $B$ band, and contains also the $B-R$ colour data of 79 galaxies.

The data are arranged in Table 4 as follows:

Column 1: The galaxy name according to Popescu et al. (1996, 1998). Isolated galaxies are designated with an asterisk.

Column 2: Effective equivalent radius in arcseconds.

Columns 3, 4: Equivalent radii in arcseconds of the 25 and $26.5 \mathrm{~B} \mathrm{mag} / \square^{\prime \prime}$ isophotes, respectively.

Column 5: Observed central surface brightness in $B$.

Column 6: Effective surface brightness in $B$, measured at $r_{\text {eff. }}$

Column \%: Total $B$-magnitude, within the $25 B \mathrm{mag} / \square^{\prime \prime}$ isophote.

Columns 8, 9: Asymptotic $B$ - and $R$-magnitudes, respectively.

Columns 10, 11: $B-R$ colour indices, measured at $r_{\mathrm{eff}}$ $\left(C I_{\text {eff }}\right)$ and at the $25 B \mathrm{mag} / \square^{\prime \prime}$ isophote $\left(C I_{25}\right)$, respectively.

Column 11a: Colour gradient, when observed in colour index profiles, is indicated as follows: "+" marks "positive" colour gradient, i.e. the galaxy is getting redder with increasing radius; "-" marks the opposite case.

Columns 12, 13: Galaxy mean minor-to-major axis ratio $(b / a)$, and position angle (PA), measured counterclockwise from North of the fitted ellipses, determined as the average between the 23 and $25 \mathrm{~B}$ mag/ $\square^{\prime \prime}$ isophotes.

Column 14: Light concentration index $c_{\text {in }}=$ $L\left(0.3 r_{25}\right) / L\left(r_{25}\right)$ introduced by Doi et al. (1993) as the ratio of the integral light contained within the isophotes with equivalent radii of $0.3 r_{25}$ and $r_{25}$, respectively.

Column 15: Light concentration index $c_{31}=$ $r\left(3 / 4 L_{\mathrm{T}}\right) / r\left(1 / 4 L_{\mathrm{T}}\right)$, defined by de Vaucouleurs (1977) as

\footnotetext{
1 Those readers who are interested in radial profile sets of all galaxies of this sample should contact the first author.
} 

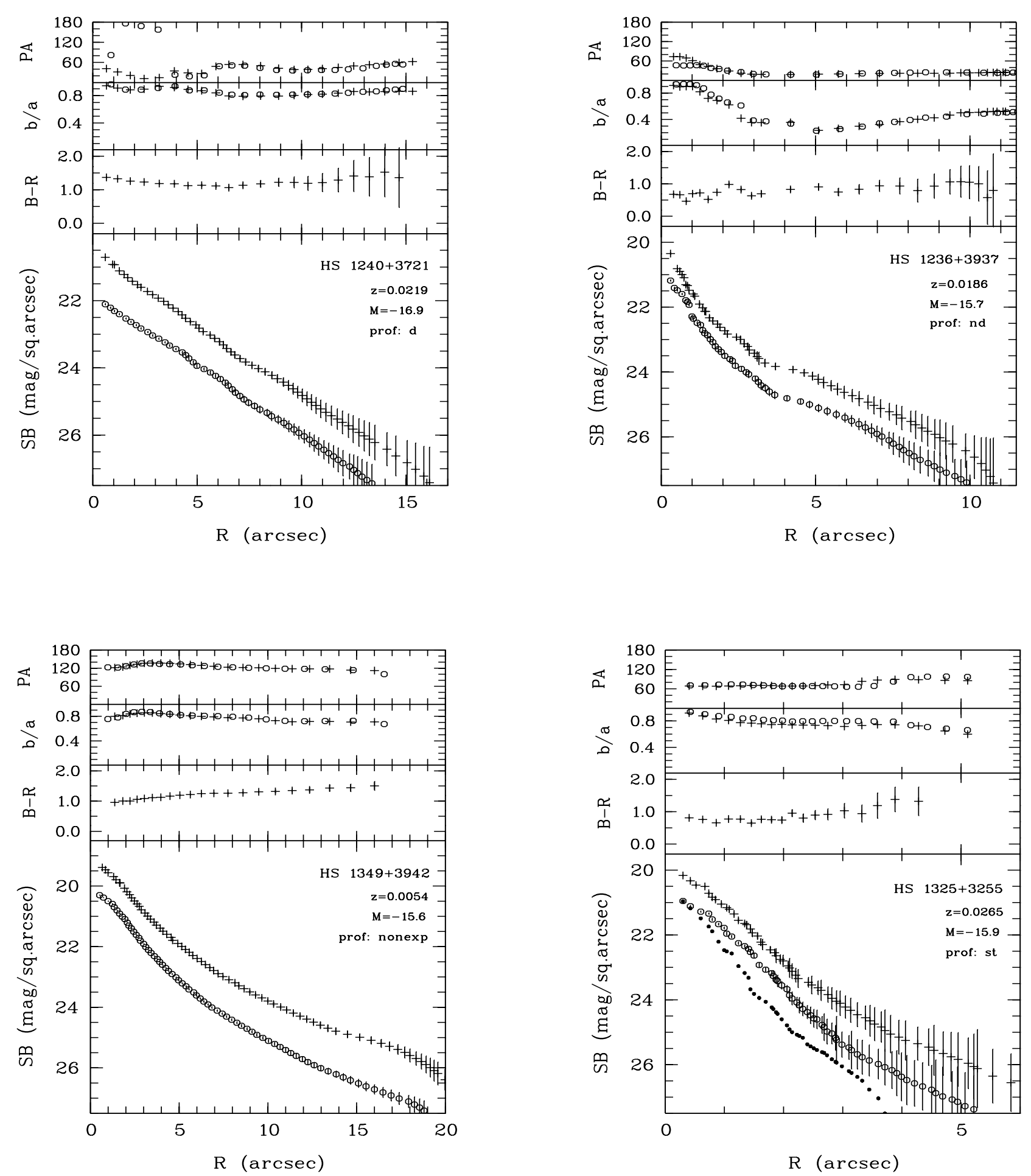

Fig. 1. Light distribution in four isolated dwarf ELGs. Surface gbrightness (SB), $(B-R)$ colour index, minor-to-major axis ratio $(b / a)$ and major axisposition angle (PA, 0 on positive $x$-axis increasing to positive $y$-axis) is shown versus the equivalent radius. Circles indicate data from $B$ passband, crosses - from $R$ passband. Profile types: HS $1240+3721$ shows a pure exponential disk (prof: d); HS 1236+3937 shows multiple components - nuclear cusp + exponential disk (prof: nd); HS 1349+3942 shows almost everywhere concave curvature, i.e. is nonexponential (prof: nonexp); HS 1325+3255 has almost stellar light profile (prof: st) - a scaled star light profile is shown by dots, for comparison 
the ratio of equivalent radii which contain a fraction $3 / 4$ and a fraction $1 / 4$ of total light, respectively.

Table 5 adds the photometric data of 16 galaxies which were observed only in $R$ band. The isophotal radii in Cols. 3, 4 refer to the $24 R \mathrm{mag} / \square^{\prime \prime}$ and $25.5 R \mathrm{mag} / \square^{\prime \prime}$ isophotes, respectively. Column 7 yields integral magnitude within $24 R \mathrm{mag} / \square^{\prime \prime}$ isophote. Geometrical parameters $(b / a)$ and $(\mathrm{PA})$ are averaged between the 22 and $24 R \mathrm{mag} / \square^{\prime \prime}$ isophotes. The concentration index in Col. $11 c_{\text {in }}=L\left(0.3 r_{24}\right) / L\left(r_{24}\right)$ refers to the $24 R \mathrm{mag} / \square^{\prime \prime}$ isophote. Other parameters are as in Table 4. Uncertain data are marked with a colon. Typical measurement errors are indicated in the last row of Table 4 . These errors are medians of the residuals of all multiple observations (in the $B$ band) of individual ELGs.

The absolute magnitudes and linear sizes of the ELGs are listed in the left part of the Tables 6 and 7 (the content of the latter is explained in Sect. 5).

In addition to the filtering of the observed frames with an adaptive smoothing filter, we performed the adaptive Laplacian filtering, too. The Laplacian filter computes the second derivative (i.e. curvature) of the SB distribution. The Laplacian filtered images do not retain any photometric information; however they are proper to disentangle the inner morphology as multiple nuclei, H II regions, spiral arms, bridges, if any. Those (faint) structures are usually hidden by large luminosity gradients of the central regions of galaxies. The Laplacian filtered images were used for the morphological classification of the ELGs (Sect. 5).

\section{The photometry errors}

The internal errors of the instrumental (non-calibrated) magnitudes and those of the particular SB profile are dominated by the error of the adopted sky background value and includes, in addition, the random count error in aperture measurements. The smooth sky background level at the position of the (masked) galaxies was computed (within PIPS) by means of an iterative filtering process which produces a "minimum surface" between the surrounding background of every particular masked object. That means, the computed sky level depends on local SB fluctuations. We measured the sky background fluctuations on the filtered CCD frames by means of small apertures in the vicinity of the studied galaxies. For the $600 \mathrm{~s}$ $B$ exposures (and $300 \mathrm{~s} R$ exposures) the sky background fluctuations are typically 0.2 percent. For the snap-shot $60 \mathrm{~s}$ exposures the corresponding fluctuations are $0.4-$ 0.8 percent in $B$ and 0.5 percent in $R$. Thus, the SB profiles are sky background limited at $\mu_{\lim } \leq 27.5 \mathrm{~B} \mathrm{mag} / \square^{\prime \prime}$ for typical $60 \mathrm{~s}$ exposures and can be traced down to $\mu_{\text {lim }} \sim 28.5 B \mathrm{mag} / \square^{\prime \prime}$ on the deepest (900 s) frames. Beyond the given limits the error in the mean sky begins to exceed the signal from the object.
Following Vader \& Chaboyer (1994) we calculated the internal errors in intensity as

$\Delta I=\sqrt{N_{\text {tot }}+\left(\delta n_{\text {sky }} A\right)^{2}}$,

where $N_{\text {tot }}$ is the total number of counts, as measured on the adaptive filtered frame within aperture $A$, which is the area between successive isophotes (in pixels), $n_{\text {sky }}$ is the mean sky counts per pixel, and $\delta$ is the fractional error in the mean sky value. This gives a signal-to-noise ratio of $\left(N_{\text {tot }}-A n_{\text {sky }}\right) / \Delta I$. The internal uncertainties are shown with error bars in Fig. 1.

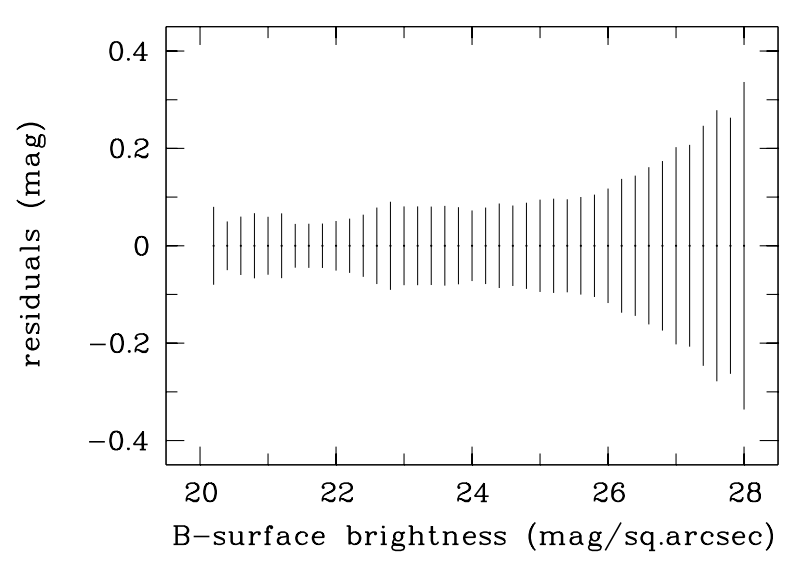

Fig. 2. The $1 \sigma$ deviations of the surface brightnesses of individual $B$ profiles from their mean values (see text) as a function of the surface brightness

Next, we checked the internal consistency of the obtained SB profiles. For a number of ELGs we obtained several frames taken during different nights or different observing sessions. Most of the repeated observations were made in $B$ passband. All these $B$ frames were processed individually. Then, the mean SB profile was calculated for a particular galaxy and the residuals of every single profile from the mean profile were computed. The $1 \sigma$ dispersion of these residuals is plotted as a function of the corresponding $B$-surface brightness in Fig. 2. This consistency test involves 13 galaxies with 30 individual SB profiles mostly extracted from $60 \mathrm{~s}$ snap-shot exposures, and 6 profiles which were obtained from $900 \mathrm{~s}$ exposures. The mean error is nearly constant at the $\sigma \sim 0.075 \mathrm{mag} / \square^{\prime \prime}$ level within $20.0 \leq \mu \leq 26.0 \mathrm{Bmag} / \square^{\prime \prime}$, followed by a rapid increase at lower surface brightnesses. At higher SB $(\mu \leq 20.0)$, which is the range of typical central surface brightnesses of ELGs, the $1 \sigma$ dispersion increases again, as an effect of varying seeing conditions during the different observing sessions.

In order to evaluate the true magnitude calibration errors we compared our total magnitude measurements with the available published data, as quoted in NASA/IPAC 
Extragalactic Database (NED). There are very few reliable photometric data available in the literature for the studied ELGs. For two galaxies, namely HS 1311+3628 (UGC 8303) and HS $1312+3508$ (UGC 8323), the $B_{\mathrm{T}}$ magnitudes are given in RC3 (de Vaucouleurs et al. 1991). For four further galaxies, (HS 1331+3906, HS 1336+3114, HS $1400+3927$ and HS 1402+3657) total magnitudes have been listed by Garnier et al. (1996), with typical uncertainties of about $0.5 \mathrm{mag}$. For the HS $1400+3927$ our total brightness is about 1 mag fainter than the one quoted by Garnier et al. (1996). The reason of this rather large difference is unclear. The remaining five common galaxies show reasonably small magnitude residuals with a mean value of $\left\langle B_{\mathrm{T} \text {,our }}-B_{\mathrm{T} \text {, cat }}\right\rangle=+0.17 \mathrm{mag}$, and an $1 \sigma$ dispersion of $0.21 \mathrm{mag}$.

Available $B$-magnitudes of the studied ELGs were collected by Popescu et al. (1996, Table 4, ( $B_{\mathrm{L}}$, Col. 7)) from various literature sources. The mean accuracy of these heterogeneous data was estimated to be of $\sim 0.5$ mag. In Fig. 3 we compare these magnitudes with our data (open circles on the plot), for 22 ELGs in common with our sample. The dispersion of individual values is large ( $1 \sigma$ $=0.78 \mathrm{mag})$. However the mean value of the magnitude residuals, $\left\langle B_{\mathrm{T} \text {,our }}-B_{\mathrm{L}}>=+0.26 \pm 0.16\right.$, is in agreement with the accuracy of the literature data, $B_{\mathrm{L}}$, and no systematic trend is evident.

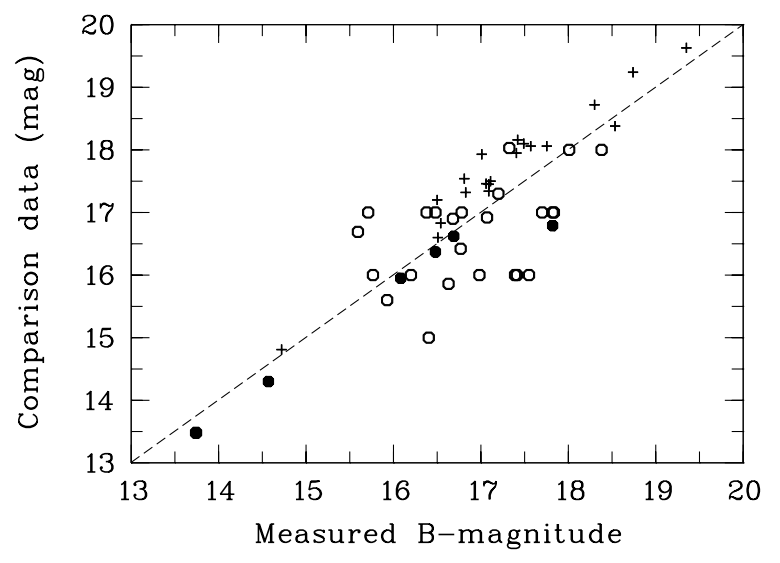

Fig. 3. Comparison of our measured $B$-magnitudes with the data obtained from literature: our $B_{\mathrm{T}}$ magnitudes versus $B_{\mathrm{T}}$ magnitudes obtained from RC3, or as quoted in Garnier et al. (1996) - filled circles (•); our $B_{25}$ magnitudes versus $m_{24.5}$, as measured by Odewahn \& Alderding (1995) on the 103a-O photographic plates - crosses (+); our $B_{\mathrm{T}}$ magnitudes versus heterogeneous magnitudes as collected by Popescu et al. (1996) from different literature sources $\left(B_{\mathrm{L}}\right)$ - open circles (o)

Further we compare our isophotal magnitudes $\left(B_{25}\right)$ with the photographic $m_{24.5}$ magnitudes measured by Odewahn \& Alderding (1995) on the blue 103a-O plates
(Fig. 3, crosses). Our $B_{25}$ magnitudes are systematically more luminous: $\left\langle B_{25}-m_{24.5, \mathrm{O}}\right\rangle=-0.42 \pm 0.06$, as could be expected because of the limiting isophote differences. However, the dispersion of individual data points is reasonably small $(1 \sigma=0.25)$, and no systematic trend is evident within the given magnitude interval. As a result of these comparisons we estimate the accuracy of our isophotal and total $B$-magnitudes to be not worse than \pm 0.2 mag.

For the $R$-magnitudes there are no comparison data available in the literature, except the photographic $m_{23.5, \mathrm{E}}$ magnitudes measured by Odewahn \& Alderding (1995) on the red 103a-E plates. In Fig. 4 our total colour indices $\left(B_{\mathrm{T}}-R_{\mathrm{T}}\right)$ are plotted versus $\left(m_{24.5, \mathrm{O}}-m_{23.5, \mathrm{E}}\right)$. Since the mean colour of ELGs is $\langle B-R\rangle \simeq 0.9$,

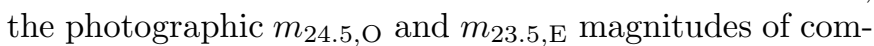
mon galaxies should refer to nearly the same aperture. Rejecting one single "discordant" photographic colour value of $m_{24.5, \mathrm{O}}-m_{23.5, \mathrm{E}}=2.34$ for $\mathrm{HS} 1325+3255$, the remaining 10 common galaxies show that our measured colours are systematically bluer by about 0.13 mag than the photographic colours ( $1 \sigma$ dispersion of $0.21 \mathrm{mag})$. The given systematics in the colour indices is probably mainly caused by differences in the CCD and photographic magnitude systems.

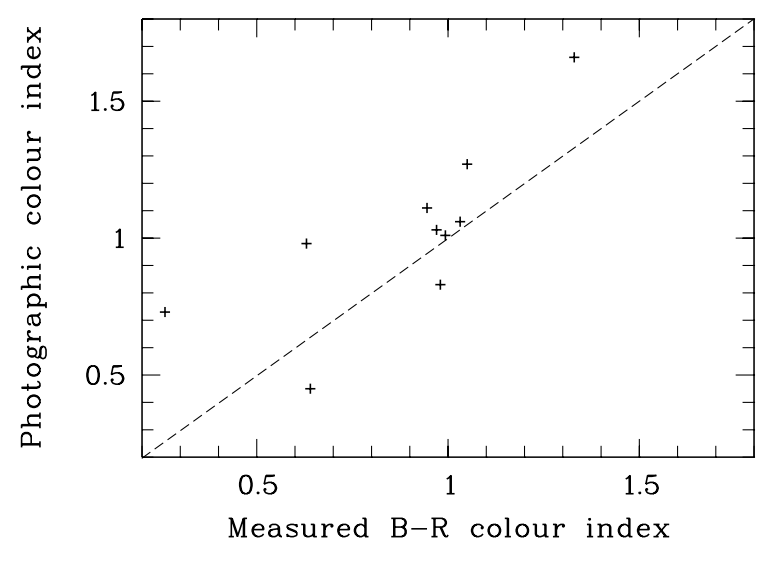

Fig. 4. Comparison of our measured total $(B-R)$ colour indices with photographic colours from Odewahn \& Alderding (1995)

\section{Morphological classification and profile fitting}

We performed the morphological classification of our sample galaxies in the framework of three classification schemes for ELGs, namely those proposed by Salzer et al. (1989, SMB89 hereafter), Loose \& Thuan (1985, LTh85 hereafter), and Telles et al. (1997, TMT97 hereafter). The scheme of SMB89 is the most quantitative one. It is based on measurable photometric parameters, as absolute 
magnitude, linear size and morphology, without making detailed use of the spectral properties.

Following the prescriptions of SMB89 we classified our ELGs as follows:

- Seyfert galaxies (Sy) and Starburst Nucleus Galaxies (SBN) have, on average, similar magnitudes $\left(M_{B} \leq-20\right)$ and sizes $(\sim 20 \mathrm{kpc})$. They can be disentangled from each other by including spectral line characteristics. The classification of a few Seyfert galaxies in our sample was obtained from the literature. Other ELGs with corresponding luminosities and sizes are classified as SBN (or as Giant Irregulars - GI, in a few cases);

- Dwarf Amorphous Nuclear Starburst Galaxies (DANS) are smaller $(\sim 10 \mathrm{kpc})$ and less luminous $\left(M_{B} \sim\right.$ -18.5) symmetric disk galaxies with bright (stellar) nuclear region, of amorphous appearance, and show no features in their disk;

- H II Hotspot galaxies (HIIH) have sizes and luminosities as DANS but are irregular in shape, with strong emission regions everywhere in the galaxy, more often found off-center;

- Dwarf HiI Hotspot galaxies (DHIIH) are as HIIH but smaller in size $(\sim 3.8 \mathrm{kpc})$ and in absolute magnitude $\left(M_{B} \sim-16.5\right)$

- Sargent-Searle (SS) objects are the smallest $(\leq 2 \mathrm{kpc})$ and the least luminous $\left(<M_{B}>=-14\right)$ ELGs;

- Interacting Pairs (IP) consist of two galaxies of comparable size, which show evidence of recent interaction.

The classes of DANS, HIIH, DHIIH and SS make up the broad group of Blue Compact Galaxies (BCGs), and most of them are also dwarfs, namely Blue Compact Dwarf Galaxies (BCDs). We assigned the general type of BCD to some less clear cases, where it was not possible to distinguish between different morphological subtypes. A few candidates in our sample were classified as Magellanic Irregulars (MI) and Giant Irregulars (GI).

The assigned morphological classes are given in Tables 6, 7 (Col. 12).

LTh85 studied a sample of $\sim 50$ BCDs on deep CCD frames and proposed a classification scheme based on the regularity of the isophotal shapes of both the highsurface-brightness (HSB) star-forming regions and the low-surface-brightness (LSB) outer region. Each BCD is described by two letters, one lower case letter which describes the location and shape of the star-forming regions ( $\mathbf{n}$ - for a single star-forming region located at the nucleus of the galaxy with circular or elliptical isophotes; $\mathbf{i}$ - for several star-forming regions which are not at the center and possess irregular isophotes), and one upper case letter which describes the shape of the outer isophotes $(\mathbf{E}-$ for circular or elliptical outer isophotes; I - for irregular outer isophotes).

LTh85 distinguished four main types of BCDs: - iE, the most common type in their sample; - nE; - iI, with subtypes of "cometary"(iI,C), and "merger"(iI,M) evi- dences; - i0, for pure stellar images without underlying older stellar population. Finally, $\sim 10$ galaxies (out of $\sim 50$ ) in LTh85 sample, which show some spiral structure, were reclassified as starburst spiral galaxies.

We classify our sample ELGs by means of above notation in Tables 6, 7 (Col. 13). For clearly non-dwarf ELGs with some spiral features we use notation S...

In their study of structural and morphological properties of H II galaxies TMT97 proposed a further morphological classification scheme, based on two main criteria: on the multiplicity of $\mathrm{H}$ II regions and on the shape of the outer isophotes. Both morphological classification schemes of LTh85 and TMT97 relay on similar structural features, but they are complementary to each other: the scheme of TMT97 specifies the multiplicity of the HII regions; LTh85 classes describe the outer isophotes in more detail. For comparison of different morphological classification schemes we assigne the "TMT97-classes" to our sample of ELGs too, by means of describing the morphology of each ELG with two integers. The first integer shows the number of resolved H II regions: 1 - single dominant giant $\mathrm{H}$ II region; 2 - double $\mathrm{H}$ II region; 3 - multiple $(\geq 3) \mathrm{H} \mathrm{II}$ region. The second integer characterizes outer structure: 1 - disturbed morphologies and irregular outer isophotes, extensions, fans or tails beyond the H II regions; 2 - symmetric and regular objects, regardless of the multiplicity of the star-forming regions (i.e. their internal structure). The assigned "TMT97-classes" are listed in Tables 6, 7 (Col. 14).

The perception of the morphology depends on redshift (and on observing conditions). TMT97 note that morphological details may be smeared out at higher redshift $(z>0.02)$, rendering the galaxies with a smoother compact appearance. In any case, the visual classification is increasingly difficult for faint and small-sized ELGs. Their poorly resolved images are dominated by bright $\mathrm{H}$ II regions, which can hide the presence of an underlying stellar component. Morphological information should be extracted using an objective classification method.

Next, we attempt to quantify the galaxian morphology by means of analysing their surface brightness profiles. Upon inspection of our SB profiles it becomes evident that many of them have linear parts and a central light excess. The occurence of an outer linear part in the SB profile was taken as an evidence for the presence of an underlying stellar disk component of the parent galaxy. We fitted the outer linear part by means of a simple exponential intensity law

$I(r)=I_{0} \exp (-\alpha r)$.

The total light emitted by the exponential disk can be computed as

$L_{\mathrm{T}}=2 \pi \alpha^{2} I_{0}$.

The excess light which remains after the subtraction of the exponential disk is often emitted by a single or multiple 
bright hotspot, which is typically offset from the geometrical center of the outer isophotes of ELGs. We refer to this excess light as to a "nucleus" which should be distinguished from the classical "bulge". The "nucleus" typically shows an unresolved "stellar" profile. The light profiles of a few luminous SBNs in the present sample show resolved central spherical components (which can usually be fitted by an $r^{1 / 4}$-profile), superimposed on the excess light of the nuclear starburst. We did not attempt to disentangle between the bulge light and the extra light emitted by the starburst.

Some SB profiles show central light depression instead of typical light excess and are more flattened than could be expected from poor seeing conditions. Flat SB profiles, which show convex curvature over linear radius, are characteristic of non-nucleated dwarf galaxies. Among the dwarf ELGs the convex SB profiles show weak starforming activity, distributed in a number of faint hotspots. A number of ELGs show nearly unresolved stellar images and their SB profiles match closely the stellar SB profiles, without any reliable underlying extended envelope. Quasi-stellar BCDs at low redshift, which appear unresolved even on deep CCD frames, are of particular interest, since they are good candidates for being truely young galaxies (see Izotov \& Thuan 1999, and references for a discussion). In addition, a few galaxies show definitely non-exponential SB profiles, i.e. they always show concave curvature over the linear radius.

The results of the profile fitting are summarized in the middle part of Tables 6,7 , with the data organised as follows:

Column 1: Galaxy name. Isolated galaxies are designated with an asterisk.

Column 2: Distance $D$ (in Mpc) calculated from the measured redshifts of Popescu et al. $(1996,1998)$, which were corrected for the Galaxy's motion with respect to the velocity centroid of the Local Group $V_{0}=v_{\odot}+300 \sin l \cos b$, (Sandage 1975) and transformed into distances assuming $H_{0}=75 \mathrm{~km} \mathrm{~s}^{-1} \mathrm{Mpc}^{-1}, q_{0}=0$.

Column 3: Absolute magnitude corrected for foreground absorption as determined by Burstein \& Heiles (1978).

Columns 4, 5: Effective $\left(r_{\text {eff }}\right)$ and isophotal $\left(r_{25}\right)$ radii in kiloparsecs.

Column 6: Seeing corrected approximate linear size of the star-forming regions $\left(r_{\mathrm{H}}\right.$ II $)$ in kiloparsecs, measured in the $B$ band as described in TMT97. $r_{\mathrm{H}}^{2}=0.25\left(F W H M_{\mathrm{H}_{\mathrm{II}}}^{2}-\right.$ $F W H M_{\mathrm{st}}^{2}$ ), where $F W H M_{\mathrm{H} \text { II }}$ is $F W H M$ of the circular area centered on the burst region of the galaxy, $F W H M_{\mathrm{st}}$ is seeing $F W H M$ of the stellar images.

Columns 7, 8: Exponential model scale length $\alpha^{-1}$ in arcseconds and in kiloparsecs, respectively.

Columns 9, 10: Exponential model central surface brightness $\mu_{0}^{\exp }$ and its corrected value $\mu_{0, \mathrm{c}}^{\exp }$, where flattening and foreground absorption corrections are applied as follows: $\mu_{0, \mathrm{c}}^{\exp }=\mu_{0}^{\exp }+2.5 \log (a / b)-$ Abs.
Column 11: Assigned profile type with following notation. Type 1: pure exponential disk - $\mathbf{d}$. Type 2: composite profile with nucleus or bulge $-\mathbf{n} / \mathbf{b}$ and disk $-\mathbf{d}$ and probably an outer halo - h:. Type 3: central light depression - $\mathbf{c}$ or always convex curvature over linear radius) - cnv. Type 4: quasi-stellar (non-resolved) profile - st. Type 5: definitely non-exponential profile with concave curvature over linear radius - cnc.

Columns 12-14: Morphological classes according to SMB89, LTh85, and TMT97, respectively, as described above.

Typical errors of the exponential disk model parameters are given in the last row of Table 6 . These errors are medians of the residuals of all multiple measurements of individual ELGs.

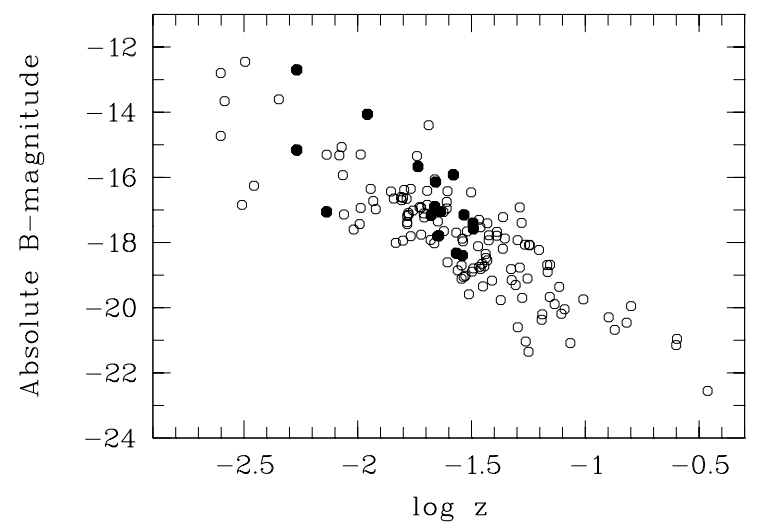

Fig. 5. Distribution of non-isolated (circles) and isolated (filled circles) ELGs at various redshifts $(z)$

\section{Statistical properties of the sample}

The studied sample of 139 ELGs (and 2 non-ELGs) was further divided into two subsamples of isolated and nonisolated galaxies, according to the clustering properties of its galaxies. The criteria for defining isolated galaxies are given in Popescu et al. (1997), based on the calculation of the nearest neighbour distances $\left(D_{\mathrm{NN}}\right)$. The $D_{\mathrm{NN}}$ represent real separations in the 3-dimensional space and are calculated (Popescu et al. 1997) as the separation between each ELG and its nearest neighbour from a comparison catalogue (ZCAT; Huchra et al. 1992; Huchra et al. 1995), for galaxies with blue magnitudes brighter than 15.5. As an example, we illustrate the case of an isolated galaxy HS $1236+3937(z=0.0184)$. It has its nearest neighbour ZCAT galaxy at a distance $8.68 h^{-1} \mathrm{Mpc}$. At this redshift the average nearest neighbour distances between the non-isolated ELGs from the field environment are $0.75 h^{-1} \mathrm{Mpc}$. Thus the galaxy HS $1236+3937$ differs 
Table 2. Comparison of mean photometric characteristics of isolated and non-isolated ELGs

\begin{tabular}{|c|c|c|c|c|c|c|c|c|c|c|c|}
\hline Sample & $\operatorname{Size}^{1)}$ & $z$ & $\begin{array}{c}M_{B} \\
{[\mathrm{mag}]}\end{array}$ & $\begin{array}{c}B_{\mathrm{T}}-R_{\mathrm{T}} \\
{[\mathrm{mag}]}\end{array}$ & $\begin{array}{c}<\mu_{\mathrm{eff}, \mathrm{c}}^{B}>^{2)} \\
{\left[\frac{\mathrm{mag}}{\mathrm{D}^{\prime \prime}}\right]}\end{array}$ & $\begin{array}{c}r_{\text {eff }} \\
{[\mathrm{kpc}]}\end{array}$ & $\begin{array}{c}r_{25} \\
{[\mathrm{kpc}]}\end{array}$ & $b / a$ & $c_{\text {in }}$ & $\begin{array}{l}\mu_{0, \mathrm{c}}^{\exp } \\
{\left[\frac{\mathrm{mag}}{\mathrm{d}^{\prime \prime}}\right]}\end{array}$ & $\begin{array}{c}\alpha^{-1} \\
{[\mathrm{kpc}]} \\
\end{array}$ \\
\hline $\begin{array}{l}\text { Non-isolated ELGs } \\
\text { (total sample) }\end{array}$ & $124(108)$ & $\begin{array}{r}0.041 \\
\pm .048\end{array}$ & $\begin{array}{c}-17.9 \\
1.8\end{array}$ & $\begin{array}{r}0.88 \\
.29\end{array}$ & $\begin{array}{r}21.6 \\
1.1\end{array}$ & $\begin{array}{l}2.1 \\
2.3\end{array}$ & $\begin{array}{l}5.0 \\
4,5\end{array}$ & $\begin{array}{r}0.71 \\
.18\end{array}$ & $\begin{array}{r}0.46 \\
.11\end{array}$ & $\begin{array}{r}21.7 \\
1.0\end{array}$ & $\begin{array}{l}1.8 \\
1.9\end{array}$ \\
\hline $\begin{array}{l}\text { Non-isolated ELGs } \\
\text { (local, } z \leq 0.033 \text { ) }\end{array}$ & $69(59)$ & $\begin{array}{r}0.018 \\
\pm .008\end{array}$ & $\begin{array}{c}-16.8 \\
1.5\end{array}$ & $\begin{array}{r}0.88 \\
.26\end{array}$ & $\begin{array}{r}21.7 \\
1.1\end{array}$ & $\begin{array}{r}1.0 \\
.6\end{array}$ & $\begin{array}{l}2.6 \\
1.5\end{array}$ & $\begin{array}{r}0.68 \\
.19\end{array}$ & $\begin{array}{r}0.48 \\
.11\end{array}$ & $\begin{array}{r}21.7 \\
1.0\end{array}$ & $\begin{array}{r}0.8 \\
.5\end{array}$ \\
\hline Isolated ELGs & $15(15)$ & $\begin{array}{r}0.021 \\
\pm .009\end{array}$ & $\begin{array}{c}-16.5 \\
1.5\end{array}$ & $\begin{array}{r}0.93 \\
.27\end{array}$ & $\begin{array}{c}22.3 \\
.7\end{array}$ & $\begin{array}{r}1.1 \\
.7\end{array}$ & $\begin{array}{l}2.5 \\
1.4\end{array}$ & $\begin{array}{r}0.68 \\
.21\end{array}$ & $\begin{array}{r}0.47 \\
.09\end{array}$ & $\begin{array}{r}22.8 \\
0.8\end{array}$ & $\begin{array}{r}1.0 \\
.5\end{array}$ \\
\hline
\end{tabular}

1) Sample size: the number of ELGs which were observed in $B$ and/or in $R$ (observed in $B$ ).

2) The mean (effective) SB within the half-light radius, corrected for the galactic absorption and for the inclination.

from its field counterparts by a factor of 12 in its clustering properties. Furthermore, the mean nearest neighbour distance for the isolated ELGs is $4.5 h^{-1} \mathrm{Mpc}$, as compared to the mean $D_{\mathrm{NN}}=1 \mathrm{~h}^{-1} \mathrm{Mpc}$ for the non-isolated ELGs. Our sample of 139 ELGs contains 15 isolated ELGs (and 2 non-ELGs), which yields a higher fraction of isolated galaxies in the present study, than in our (earlier) photometric study of faint galaxies in voids (Vennik et al. 1996).

Next we derive mean photometric characteristics of the studied sample and search for possible differences between the mean parameters of the isolated and non-isolated ELGs in Table 2 and in Figs. 5-7. Standard mean photometric parameters are evaluated in the $B$ band. For the 16 ELGs which were observed only in the $R$ band, their $R$ magnitudes were transformed into $B$ assuming an average colour of $B-R=0.9$ (which is valid for our sample, see below). Those transformed data are included in Figs. 5, 6 . They do not show any systematic deviations. Figure 5 demonstrates some bias effects in our sample. While all our ELGs are distributed up to $z \simeq 0.34$, the isolated ELGs are detected within $z \leq 0.033$, only. The available redshift catalogs do not permit us to evaluate true isolation properties of the ELGs at greater distances (see Popescu et al. 1997 for a complete discussion). Hereafter we call the volume within $z \leq 0.033$ the "local volume". The upper boundary of the absolute magnitude - $\log z$ relation in Fig. 5 corresponds to the limiting apparent magnitude of about 19.5, which yields the formal completeness limit within the "local volume" of $M_{B} \leq-16.3$. However, the initial sample of ELGs was not selected as a typical continuum magnitude limited sample, rather as an emission-line flux limited sample, as described in Popescu et al. (1997).

Table 2 summarizes the mean parameters in $B$ band and their errors of three subsamples of ELGs. The characteristics of the total sample of 124 non-isolated ELGs are given in the first row. The second and third rows of the Table 2 present the mean parameters of the nonisolated and isolated ELGs, within similar distance limits, i.e. within "local volume".

Figures 6a-d show different correlations. In these figures the isolated resolved ELGs are presented by filled circles; non-isolated resolved ELGs - by open circles. The ELGs having almost stellar images are shown by crosses, in order to disentangle the possible seeing effects.

The analysis of the tabulated and plotted data leads to the following results:

The ELGs of our sample are distributed within a broad interval of distances (up to $z \simeq 0.34$ ), of absolute magnitudes $\left(-22.5 \leq M_{B} \leq-12.5\right)$, and of radii $\left(0.2 \leq r_{25} \leq 17.9 \mathrm{kpc}\right)$, and consist of almost all varieties of actively star-forming galaxies, as classified in SMB89, from the faintest Sargent-Searle dwarfs to the giant SBNs and include even a few AGNs.

Within the "local volume" where the isolation properties could have been evaluated, both the isolated and non-isolated ELGs show similar mean values of their total luminosities and radii, with those two parameters being distributed within $-19.6 \leq M_{B} \leq-12.5$ and $0.2 \leq r_{25} \leq$ $6.3 \mathrm{kpc}$, respectively. According to their photometric and morphological characteristics the ELGs within "local volume" belong to the broad class of BCGs, with most of them being dwarfs (i.e. BCDs).

Figures $6 \mathrm{a}$ and $6 \mathrm{c}$ show the distributions of the two different SB characteristics of our sample galaxies. Figure $6 \mathrm{a}$ presents the mean effective $\mathrm{SB}\left(<\mu_{\mathrm{eff}, \mathrm{c}}^{B}>\right)$, which is calculated as a mean SB within the half-light radius and is corrected for the inclination and for the galactic absorption as taken from the NED. This SB parameter includes both the light of the $\mathrm{H}$ II regions and that of the underlying parent galaxy. As could be expected, the quasi-stellar ELGs with almost unresolved images yield the highest SB (crosses in Fig. 6). The mean effective SB of the studied ELGs appears to have a lower bound at $\sim 24 B \mathrm{mag} / \square^{\prime \prime}$. They are distributed over 4 magnitudes in SB. Remarkably, there 

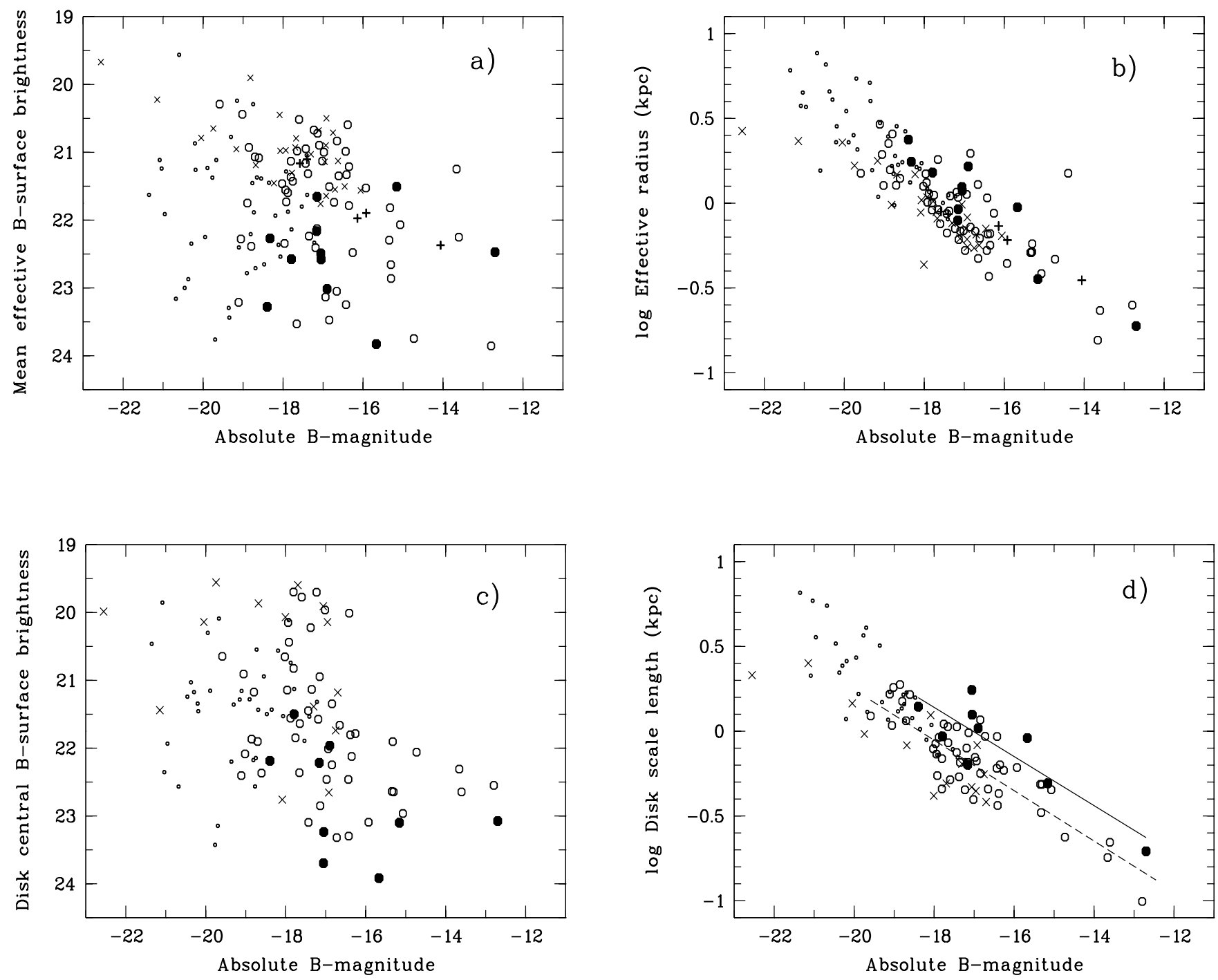

Fig. 6. Relations between the surface brightness characteristics: a) mean (effective) surface brightness within the half-light radius, b) log effective radius, c) exponential disk central surface brightness, d) log exponential disk scale length versus absolute B-magnitude. Big circles (০) mark non-isolated resolved ELGs in "local volume", filled circles (•) mark isolated resolved ELGs in the same volume. Small circles mark distant ELGs (outside the "local volume"). Unresolved ELGs with quasi-stellar images are marked by $\times$ - for non-isolated ELGs and by +- for isolated ELGs. d) shows the linear fit of log disk-scale-length - absolute magnitude relation for isolated (continuous line) and non-isolated (dashed line) ELGs within the "local volume"

are intrinsically luminous (giant) ELGs with low SB, too. Probably, those ELGs have smaller H II regions than those found in SBNs and could be classified as Giant Irregulars (GI). The isolated ELGs show marginally fainter mean SB $\left(22.3 \pm 0.7 B \mathrm{mag} / \square^{\prime \prime}\right)$ than the non-isolated (and non-stellar) ELGs $\left(21.7 \pm 1.1 B \mathrm{mag} / \square^{\prime \prime}\right)$, but the isolated ELGs avoid the brightest 2 mag in SB covered by the non-isolated ELGs.

Figure 6c shows the distribution of another SB parameter - the central SB of the exponential disk component $\left(\mu_{0, c}^{\exp }\right)$, with the same corrections applied as for the mean effective SB (see above). This parameter characterizes the underlying parent galaxy. Naturally, the SB profiles of the 33 ELGs, which are either quasi-stellar or show always convex or concave curvature over linear radius, could not be fitted by the exponential disk model. Further 14 ELGs with quasi-stellar images provide a poor fit with exponential model. Those ELGs are marked with crosses in Figs. 6c and $6 \mathrm{~d}$. Two features could be noted in the distribution of the disk central SB:

1) A conspicuous empty area in the upper right part of Fig. 6c, with the parametric limits: $M_{B}>-16.5$ and $\mu_{0, \mathrm{c}}^{\exp }<21.6$. That means, all dwarf ELGs with $M_{B}>$ -16.5 have dimmer disks than the typical Freeman's disk 
$\left(\mu_{0}^{\exp }=21.6\right)$. The disks of intrinsically brighter ELGs with $M_{B}<-16.5$ are scattered within 4 mag around and below of the Freeman's disk typical SB.

2) Isolated ELGs tend to have lower underlying disk central SB $\left(<\mu_{0, \mathrm{c}}^{\exp }>=22.8 \pm 0.8\right)$, compared to the nonisolated ELGs of the same $M_{B}(21.7 \pm 1.0)$.

Figures $6 \mathrm{~b}, \mathrm{~d}$ show that ELGs are confined into a narrow strip in $\log r-M_{B}$ plane. The isolated ELGs tend to have larger linear extent than the non-isolated ELGs of the same luminosity. This tendency becomes more evident for the exponential disk model scale lengths. In Fig. $6 \mathrm{~d}$ we perform a linear fit to the log disk-scale-length $-M_{B}$ relation for both the isolated and the non-isolated ELGs within the "local volume". The results show that the isolated ELGs possess underlying disks which are, on average, about a factor of 1.8 larger than the disks of the non-isolated ELGs. This difference appears statistically significant at the $1.75 \sigma$ level.

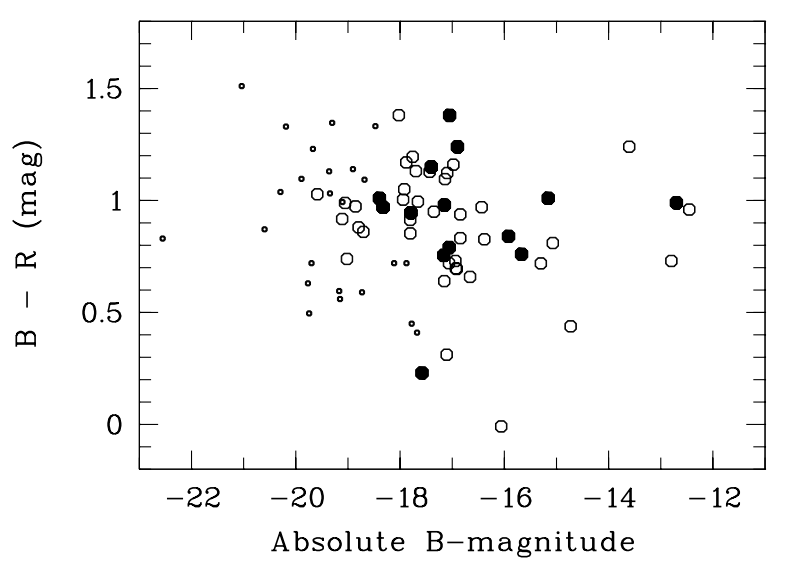

Fig. 7. The total $(B-R)$ colour indices versus absolute $B$-magnitude of non-isolated (circles) and isolated (filled circles) ELGs

Both the isolated and non-isolated ELGs show blue mean colour of $\left\langle B_{\mathrm{T}}-R_{\mathrm{T}}\right\rangle=0.9 \pm 0.3$ (Fig. 7). We scrutinized the radial colour index (CI) profiles for the occurence of colour gradients in the range of high signalto-noise ratio, which typically extends up to the $26 \mathrm{~B}$ $\mathrm{mag} / \square^{\prime \prime}$ isophote, but excluding the very nuclear region within a few arcseconds, where the colours can be affected by changing seeing conditions and/or the occurence of a single luminous (blue) H II region. Extranuclear colour changes are determined both by the stellar populations of the parent galaxy and by further H II knots, when distributed outside of circumnuclear region. We did not attempt to measure the exact values of colour gradients, but classified them (in Table 4, Col. 11a) as follows: definitely positive, i.e. with blue center and getting redder outwards $(+)$, probably positive $(+:)$, definitely negative $(-)$, prob-

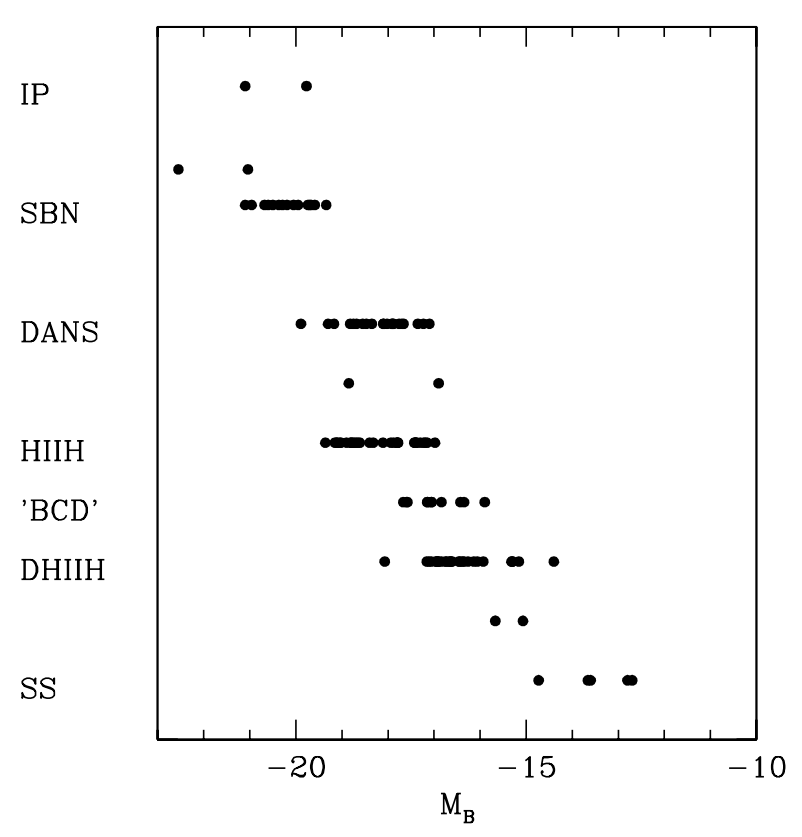

Fig. 8. The morphological classes of SMB89 versus absolute $B$-magnitude

ably negative (-:) and no gradients (blank). Nearly half of the determined $B-R$ colour index profiles show reliable radial colour changes.

We searched for possible correlations of the colour gradients with different photometric and morphological characteristics, with the following results:

1) The colour gradients are not correlated with the integral colours.

2) The colour gradients are marginally correlated with the absolute $B$-magnitude in the sense that intrinsically fainter ELGs tend to show a larger fraction of positive gradients (all dwarf ELGs with $M_{B}>-16.5$ show positive gradients, if any).

3) The colour gradients are marginally correlated with the SB profile type: pure exponential disks (type 1) and disks with central flattening (type 3 ) show negative gradients, if any; definitely non-exponential profiles (type 5) show positive gradients, if any; among the multiple component profiles (type 2) the positive colour gradients clearly dominate; unresolved quasi-stellar profiles (type 4) typically do not reveal any reliable colour changes, as could be expected.

4) Among the isolated ELGs there is a slightly higher fraction of positive gradients, which is related to the higher fraction of non-exponential SB profiles among the isolated ELGs (see next paragraph).

The results of SB profile type classification are summarized in Table 3. Of our galaxies, $73 \%$ have SB profiles with some disk component. Nearly half of those disks are 
superposed with extra light emitted by bulges or nuclei (i.e. by bright $\mathrm{HII}$ regions). About $25 \%$ of our ELGs show unresolved images and their SB profiles could not have been disentangled from stellar light profiles. The isolated ELGs show slightly higher fraction of unresolved images and non-exponential light profiles, compared to nonisolated ELGs. But the difference is not statistically significant, because of the small number of isolated ELGs. Only for 8 of the 15 isolated ELGs an underlying disk component have been discerned in their SB profiles.

The three different morphology classifications presented in Tables 6,7 will be discussed in detail in a separate paper. A "snapshot" statistics shows that about $83 \%$ of our ELGs belong to the various subtypes of BCGs, which include many subclasses of intrinsically faint ELGs, as discerned by SMB89 in their quantitative classification scheme. The remaining 17\% ELGs are classified as giant SBN, with several of them possessing AGN features, or as interacting pairs (IP). Figure 8 shows the distribution of the absolute blue magnitude with the classification of SBM89. To establish this graph, we assigned numerical values to the classification scheme, namely 1 for SS, 2 for DHIIH, 3 for HIIH, 4 for DANS, 5 for SBN, and 6 for IP. Uncertain classification between two classes were assigned the average value, e.g. DHIIH/HIIH got a 2.5. Those galaxies with the rough classification "BCD" were also assigned to the value 2.5. As can be expected from the defination of the classification system, the absolute magnitude correlates with the classification. But the luminosity distribution within one class is rather broad, up to 4 mag peak-to-peak. This scatter reflects both, the intrinsic distribution of luminosities within each class (Salzer et al. 1989, Fig. 9a) and the uncertainties of our classification process.

TMT97 quantify the multiplicity of the H II regions and describe the shape of the outer isophotes. According to the TMT97-scheme $72 \%$ of the studied ELGs possess a single nucleus and $28 \%$ show the presence of double or multiple nuclei. The second parameter in the TMT97scheme, describing the outer structure, yields similar frequencies: $71 \%$ of the ELGs are symmetric and regular objects, regardless of the multiplicity of their $\mathrm{H}$ II regions, and $29 \%$ show disturbed outer isophotes.

LTh85 classified the morphology of the BCDs in more qualitative terms based mostly on the regularity both of the center and of the outskirts of the galaxies. Considering here the appearance of the bright central region we assign $39 \%$ of the ELGs into class $\mathbf{n}$ (single SFR and regular isophotes), $52 \%$ of the ELGs into class i (several SFR and irregular isophotes), The remaining $9 \%$ of the ELGs are (non-dwarf) distant spiral galaxies of the types SBN, AGN, and a few interacting pairs.
Table 3. Comparison of the SB profile type frequencies of ELGs

\begin{tabular}{l|cc|cc}
\hline Profile description (prof. type) & \multicolumn{2}{|c|}{ non-isolated } & \multicolumn{2}{|c}{ isolated } \\
& $\mathrm{Nr}$ & $\%$ & $\mathrm{Nr}$ & $\%$ \\
\hline & & & & \\
Pure exponentials (1 d) & 27 & 22 & 2 & 13 \\
Multiple components (2 b/nd) & 46 & 37 & 5 & 33 \\
(disk+bulge/nucleus) & & & & \\
Central light depression (3 cnv) & 17 & 14 & 1 & 7 \\
Unresolved (4 st) & 27 & 22 & 4 & 27 \\
Non-exponentials (5 cnc) & 7 & 5 & 3 & 20 \\
\hline
\end{tabular}

\section{Summary of the results}

The dedicated search for ELGs on the blue objective prism plates revealed a large number of intrinsically faint galaxies, and with small linear sizes. Surface photometry of a subsample of 139 ELGs show that nearly $25 \%$ of them have almost unresolved stellar images and quasi-stellar SB profiles. Those unresolved galaxies could not have been separated from stars by traditional morphological surveys.

Among the studied ELGs there is a higher percentage of isolated galaxies than in the study of Vennik et al. (1996). The 15 isolated ELGs are intrinsically faint and small ELGs. Isolated ELGs show, consequently, a slightly higher fraction of quasi-stellar SB profiles.

SB profile decomposition into disk and excess light components has been performed for most but the unresolved ELGs. ELGs show more SB profiles with (central) light excess, when compared to those in the morphological surveys, because of the bright HII regions in the ELGs. Among the resolved dwarf ELGs $\left(M_{B} \geq-16.5\right)$ an underlying stellar disk component has been detected for most of them, at least marginally. The underlying disks of the isolated ELGs are of lower SB and have larger scale lengths than the non-isolated ELGs of the same luminosity. Consiquently, the isolated galaxies could have lower stellar (i.e. baryonic) density than the clustered galaxies. For a full discussion of this issue the baryonic content in gaseous $(\mathrm{HI})$ form has to be considered, and recent studies (Hopp 1998) suggest that this content might be unsually high in these galaxies.

The observed ELGs are blue in $B-R$, which should be expected since the objects were selected from blue plates. Our small sample does not reveal any significant colour differences between isolated and non-isolated ELGs. Nearly half of the sample shows reliable radial colour changes. The frequency of raising outwards and decreasing outwards colour index profiles is nearly equal, but depends slightly on the intrinsic luminosity and on the profile type (i.e. morphology) of the ELGs.

The preliminary results concerning the (marginally detected) underlying stellar components in the dwarf ELGs should be confirmed by measurements on deeper frames, obtained in good seeing conditions. 
A detailed comparison to the results of other photometric studies of dwarf and/or emission line galaxies like e.g. Salzer et al. (1989), Telles et al. (1997), Vader \& Chaboyer (1994), Vitores et al. (1996), Cruzen et al. (1997) will be postponed to a main journal paper.

Acknowledgements. We would like to thank Dr. A.P. Fairall for the careful review of this manuscript. We gratefully acknowledge Prof. H. Elsässer for interesting discussions and Dr. G.M. Richter for using his software package. J.V. acknowledges the financial support and hospitality of the Max-Planck-Institute for Astronomy during several visits. This research has made use of the NASA/IPAC Extragalactic Database (NED) which is operated by the Jet Propulsion Laboratory, California Institute of Technology, under contract with the National Aeronautics and Space Administration.

\section{References}

Bender R., Möllenhoff C., 1987, A\&A 177, 71

Burstein D., Heiles C., 1978, ApJ 225, 40

Christian C.A., Adams M., Barnes J.V., Butcher H., Mould J.R., Siegel M., 1985, PASP 97, 363

Cruzen S.T., Weistrop D., Hoopes C.G., 1997, AJ 113, 1983

Dekel A., Silk J., 1986, ApJ 303, 39

de Vaucouleurs G., de Vaucouleurs A., Corwin H.G., 1976, Second Reference Catalogue of Bright Galaxies. Univ. of Texas Press, Austin (RC2)

de Vaucouleurs G., 1977, in: "Evolution of Galaxies and Stellar Populations", Larson R. and Tynsley B. (eds.). Yale Univ. Observatory, New Haven, p. 43

de Vaucouleurs G., de Vaucouleurs A., Corwin H.G. Jr., Buta R.J., Paturel G., Fouque P., 1991, Third Reference Catalogue of Bright Galaxies. Springer-Verlag, New-York (RC3)

Doi M., Fukugita M., Okamura S., 1993, MNRAS 264, 832

Garnier R., Paturel G., Petit C., Marthinet M.C., Rousseau J., 1996, A\&AS 117, 467

Hopp U., Kuhn B., Thiele U., Birkle K., Elsässer H., 1995,
A\&AS 109, 537

Hopp U., 1998, in: "The Evolving Universe", Hamilton D. (ed.). Kluwer, p. 59

Huchra J., Geller M., Clemens C., Tokarz S., Michel A., 1992, Bull. CDS 41, 31

Huchra J., Geller M.J., Clemens C.M., Tokarz S.P., Michel A., 1995, The Center for Astrophysics Redshift Catalogue, electronic version

Izotov Y.I., Thuan T.X., 1999, ApJ 511, 639

Kuhn B., Hopp U., Elsässer H., 1997, A\&A 318, 405

Loose H.-H., Thuan T.X., 1985, in: "Star-Forming Dwarf Galaxies and Related Objects", Kunth D., Thuan T.X. and Van T.T. (eds.). Paris: Éditions Frontières, p. 73 (LTh85)

Lorenz H., Richter G.M., Capaccioli M., Longo G., 1993, A\&A 277,321

Odewahn S.C., Alderding G., 1995, AJ 110, 2009

Popescu C.C., Hopp U., Hagen H.J., Elsässer H., 1996, A\&AS 116,43

Popescu C.C., Hopp U., Elsässer H., 1997, A\&A 328, 756

Popescu C.C., Hopp U., Hagen H.J., Elsässer H., 1998, A\&AS 133,13

Salzer J., McAlpine G.M., Boroson T.A., 1989, ApJS 70, 479 (SMB89)

Sandage A., 1975, Galaxies and the Universe. University of Chicago press

Stickel M., Fried J.W., Kúhr H., 1993, A\&AS 98, 393

Szomoru A., van Gorkom J.H., Gregg M.D., Strauss M.A., 1996, AJ 111, 2150

Telles E., Melnik J., Terlevich R., 1997, MNRAS 288, 78 (TMT97)

Vader J.P., Chaboyer B., 1994, AJ 108, 1209

Vennik J., Hopp U., Kovachev B., Kuhn B., Elsässer H., 1996, A\&AS 117, 261

Vitores A.G., Zamorano J., Rego M., Gallego J., Alonso O., 1996, A\&AS 120, 385

Weistrop D., Hintzen P., Kennicutt Jr. R.C., Lin C., Lowenthal J., Cheng K.-P., Oliversen R., Woodgate B., 1992, ApJ 396, L23

Weistrop D., Hintzen P., Liu C., Lowenthal J., Cheng K.-P., Oliversen R., Brown L., Woodgate B., 1995, AJ 109, 981 
Table 4. Observed model-free photometric data in $B$ and colour characteristics

\begin{tabular}{|c|c|c|c|c|c|c|c|c|c|c|c|c|c|c|}
\hline Galaxy & $\begin{array}{c}r_{\mathrm{eff}} \\
{\left[{ }^{\prime \prime}\right]}\end{array}$ & $\begin{array}{c}r_{25} \\
{\left[{ }^{\prime \prime}\right]}\end{array}$ & $\begin{array}{c}r_{26.5} \\
{\left[^{\prime \prime}\right]}\end{array}$ & $\begin{array}{c}\mu_{0}^{B} \\
{\left[\frac{\mathrm{mag}}{\square^{\prime \prime}}\right]}\end{array}$ & $\begin{array}{c}\mu_{\mathrm{eff}}^{B} \\
{\left[\frac{\mathrm{mag}}{\square^{\prime \prime}}\right]}\end{array}$ & $\begin{array}{c}B_{25} \\
{[\mathrm{mag}]}\end{array}$ & $\begin{array}{c}B_{\mathrm{T}} \\
{[\mathrm{mag}]}\end{array}$ & $\begin{array}{c}R_{\mathrm{T}} \\
{[\mathrm{mag}]}\end{array}$ & $\begin{array}{c}C I_{\text {eff }} \\
{[\mathrm{mag}]}\end{array}$ & $\begin{array}{l}C I_{25} \\
{[\mathrm{mag}]}\end{array}$ & $b / a$ & $\begin{array}{l}\mathrm{PA} \\
{\left[{ }^{\circ}\right]}\end{array}$ & $c_{\text {in }}$ & $c_{31}$ \\
\hline$(1)$ & $(2)$ & $(3)$ & $(4)$ & $(5)$ & $(6)$ & $(7)$ & $(8)$ & $(9)$ & $(10)$ & $(11)$ & $(12)$ & $(13)$ & $(14)$ & $(15)$ \\
\hline HS $0731+6348$ & 1.3 & 3.4 & 5.1 & 20.5 & 21.20 & 18.38 & 18.32 & 17.49 & 0.6 : & $1.20+:$ & 0.80 & 44 & 0.46 & 2.30 \\
\hline HS $0732+6503$ & 3.2 & 8.2 & 13.0 & 20.8 & 22.07 & 17.02 & 16.90 & 15.52 & 1.36 & $1.15-$ & 0.74 & 44 & 0.49 & 2.76 \\
\hline HS $0749+5649$ & 1.8 & 5.4 & 7.7 & 20.4 & 21.46 & 17.71 & 17.64 & 16.94 & 0.48 & $0.90+:$ & 0.80 & 8 & 0.57 & 2.64 \\
\hline HS $0752+5603$ & 3.3 & 10.7 & 15.3 & 20.0 & 22.08 & 16.59 & 16.48 & 15.51 & 0.92 & 0.99 & 0.90 & 47 & 0.58 & 3.87 \\
\hline HS $0757+6441$ & 2.1 & 6.0 & 7.9 & 20.7 & 21.71 & 17.61 & 17.55 & 16.45 & 1.05 & $0.99-:$ & 0.66 & 50 & 0.48 & 2.50 \\
\hline HS $0805+5742$ & 1.9 & 5.2 & 7.4 & 20.3 & 21.47 & 17.65 & 17.59 & 16.46 & 1.05 & $0.94-:$ & 0.83 & 6 & 0.50 & 2.51 \\
\hline HS $0831+6215$ & 1.6 & 4.8 & 6.9 & 20.2 & 21.24 & 17.68 & 17.62 & 16.93 & 0.70 & 0.89 & 0.95 & 73 & 0.53 & 2.55 \\
\hline HS $0915+5540$ & 2.0 & 5.9 & 8.3 & 20.3 & 21.32 & 17.32 & 17.25 & 15.90 & 1.28 & 1.27 & 0.74 & 59 & 0.54 & 2.64 \\
\hline HS $1222+3741$ & 1.2 & 3.2 & 4.6 & 19.7 & 21.00 & 18.46 & 18.40 & 17.99 & $0.45:$ & $0.3:$ & 0.85 & 16 & 0.40 & 2.23 \\
\hline HS $1223+3938$ & 3.1 & 7.9 & 10.5 & 20.1 & 22.22 & 17.15 & 17.07 & 16.48 & 0.62 & $0.38-$ & 0.63 & 50 & 0.42 & 2.85 \\
\hline HS $1226+3620$ & 2.8 & 5.3 & 7.4 & 22.1 & 23.31 & 18.69 & 18.50 & & & & 0.78 & 178 & 0.31 & 2.60 \\
\hline HS $1226+3719^{*}$ & 1.7 & 3.3 & 6.3 & 21.0 & 23.32 & 19.50 & 19.18 & & & & 0.92 & 60 & 0.49 & 4.13 \\
\hline HS $1232+3609$ & 1.9 & 4.3 & 5.6 & 21.2 & 23.44 & 19.21 & 19.07 & & & & 0.64 & 161 & 0.44 & 3.74 \\
\hline HS $1232+3612$ & 3.6 & 9.5 & 16.5 & 19.6 & 22.08 & 16.54 & 16.39 & 15.76 & 0.67 & 0.67 & 0.70 & 74 & 0.50 & 3.21 \\
\hline HS $1232+3846$ & 6.5 & 11.6 & 17.3 & 20.5 & 23.85 & 17.20 & 16.93 & 16.21 & 0.79 & $0.70-$ & 0.40 & 162 & 0.38 & 3.29 \\
\hline HS $1232+3947^{*}$ & 2.1 & 5.9 & 8.3 & 20.5 & 21.68 & 17.55 & 17.47 & 16.72 & 0.71 & $0.93+$ & 0.34 & 78 & 0.49 & 2.68 \\
\hline HS $1236+3821^{*}$ & 8.9 & 19.7 & 37.2 & 19.5 & 23.47 & 15.64 & 15.31 & 14.52 & 0.86 & 0.80 & 0.65 & 50 & 0.55 & 6.50 \\
\hline HS $1236+3937^{*}$ & 2.8 & 4.4 & 8.4 & 21.2 & 24.11 & 19.16 & 18.68 & 17.92 & 0.73 & $0.88+$ & 0.40 & 115 & 0.45 & 4.32 \\
\hline HS $1240+3721^{*}$ & 4.2 & 7.3 & 11.3 & 22.1 & 23.54 & 18.07 & 17.82 & 16.58 & 1.14 & 1.14 & 0.85 & 126 & 0.30 & 2.64 \\
\hline HS $1240+3755$ & 3.1 & 8.2 & 11.2 & 20.1 & 21.7: & 16.7: & 16.6: & & & & 0.69 & 131 & 0.44 & 2.68 \\
\hline HS $1242+3551$ & 2.0 & 3.9 & 5.6 & 21.8 & 23.08 & 19.22 & 19.06 & & & & 0.73 & & 0.28 & 2.37 \\
\hline HS $1244+3648$ & 1.3 & 4.5 & 7.3 & 19.6 & 20.60 & 17.67 & 17.58 & & & & & & 0.64 & 2.95 \\
\hline HS $1254+3323$ & 0.8 & 2.8 & 4.0 & 19.3 & 20.27 & 18.20 & 18.17 & 17.21 & 0.96 & 1.01 & 0.89 & 56 & 0.60 & 2.44 \\
\hline HS $1255+3506$ & 2.2 & 6.3 & 9.9 & 20.2 & 21.83 & 17.49 & 17.37 & & & & 0.72 & 59 & 0.54 & 2.89 \\
\hline HS $1256+3505$ & 1.7 & 7.0 & 10.5 & 19.4 & 20.88 & 17.01 & 16.94 & & & & 0.70 & 40 & 0.70 & 3.36 \\
\hline HS $1258+3438$ & 2.5 & 4.7 & 7.7 & 21.9 & 23.16 & 18.83 & 18.59 & & & & 0.46 & 26 & 0.35 & 2.77 \\
\hline HS $1301+3209$ & 2.3 & 5.1 & 7.5 & 21.5 & 22.17 & 18.00 & 17.90 & 17.18 & 0.72 & 0.80 & 0.84 & 36 & 0.33 & 2.28 \\
\hline HS $1301+3312$ & 1.3 & 3.7 & 5.2 & 20.5 & 21.47 & 18.53 & 18.45 & & & & 0.83 & 21 & 0.50 & 2.58 \\
\hline HS $1301+3325$ & 1.6 & 4.5 & 6.9 & 20.4 & 21.65 & 18.10 & 18.02 & & & & 0.85 & 178 & 0.51 & 2.74 \\
\hline HS $1302+3046$ & 6.7 & 13.0 & 16.5 & 21.6 & 23.06 & 16.51 & 16.42 & 15.39 & 0.96 & 1.13 & 0.38 & 3 & 0.27 & 2.35 \\
\hline HS $1304+3529$ & 3.0 & 8.5 & 11.6 & 20.0 & 21.92 & 16.83 & 16.75 & 16.45: & 0.25 : & $0.7:$ & 0.77 & 4 & 0.51 & 3.00 \\
\hline HS $1306+3320 *$ & 3.7 & 9.2 & 14.0 & 20.2 & 22.70 & 17.06 & 16.87 & 15.90 & 1.00 & 1.05 & 0.53 & 10: & 0.49 & 3.59 \\
\hline HS $1306+3525$ & 3.8 & 9.3 & 14.0 & 20.9 & 22.58 & 17.09 & 16.93 & & & & 0.56 & 50 & 0.49 & 3.06 \\
\hline HS $1306+3527$ & 2.8 & 7.5 & 10.0 & 20.3 & 22.37 & 17.41 & 17.32 & & & & 0.75 & 136 & 0.47 & 3.44 \\
\hline HS $1308+3044$ & 2.7 & 7.8 & 11.1 & 20.2 & 21.52 & 16.81 & 16.74 & 15.69 & 0.98 & $1.00-:$ & 0.41 & 145 & 0.49 & 2.60 \\
\hline HS $1309+3409$ & 2.5 & 6.9 & 10.3 & 20.5 & 22.14 & 17.42 & 17.30 & 15.97 & 1.20 & $1.14-$ & 0.77 & 15 & 0.50 & 3.00 \\
\hline HS $1311+3638$ & 31.3 & 51.8 & 76.0 & 20.9 & 23.78 & 13.97 & 13.74 & 12.91 & 0.83 & $0.76-:$ & 0.78 & 172 & 0.27 & 2.50 \\
\hline HS $1312+3112$ & 4.1 & & 6.1 & 24.7 & 25.6: & & $20.2:$ & & & & 0.26 & 20 & 0.12 & 2.14 \\
\hline HS $1312+3508$ & 12.5 & 27.7 & 43.0 & 18.5 & 22.77 & 14.72 & 14.57 & & & & 0.66 & 98 & 0.42 & 3.16 \\
\hline HS $1312+3847$ & 1.8 & 5.2 & 8.2 & 20.5 & 21.73 & 17.96 & 17.81 & & & & 0.59 & 85 & 0.57 & 2.90 \\
\hline HS $1314+3320$ & 1.0 & 2.3 & 3.5 & 21.1 & 22.3: & 19.8: & 19.7: & & & & 0.83 & 121 & 0.42 & 2.69 \\
\hline HS $1315+3132$ & 1.3 & 3.0 & 5.0 & 21.1 & 22.34 & 19.22 & 19.06 & & & & & & 0.43 & 2.69 \\
\hline HS $1318+3239$ & 1.6 & 3.5 & 5.3 & 21.5 & 22.53 & 19.14 & 19.00 & & & & 0.59 & 126 & 0.37 & 2.55 \\
\hline HS $1318+3406$ & 2.8 & 6.6 & 8.4 & 20.9 & 21.75 & 17.15 & 17.11 & & & & 0.85 & 165 & 0.34 & 2.24 \\
\hline HS $1319+3224$ & 1.5 & 3.6 & 5.8 & 21.1 & $22.7:$ & 19.2: & 19.0: & & & & 0.66 & 27 & 0.46 & 3.10 \\
\hline HS $1325+3225$ & 1.5 & 3.8 & 5.4 & 21.0 & 22.1: & 18.7: & 18.6: & & & & & & 0.44 & 2.67 \\
\hline HS $1325+3255^{*}$ & 1.3 & 2.9 & 4.3 & 20.9 & 22.36 & 19.35 & 19.21 & 18.37 & 0.8: & $0.9:+$ : & 0.80 & 158 & 0.42 & 2.60 \\
\hline HS $1327+3126$ & 1.1 & 3.0 & 4.5 & 20.6 & 21.44 & 18.83 & 18.74 & & & & 0.82 & 40 & 0.45 & 2.41 \\
\hline HS $1327+3412$ & 1.2 & 3.0 & 4.7 & 20.6 & 21.8: & 19.0: & 18.9: & & & & & & 0.47 & 2.77 \\
\hline HS $1327+3749$ & 2.0 & 4.5 & 6.4 & 21.2 & 22.7: & 18.8: & 18.6: & & & & 0.56 & 160 & 0.39 & 2.81 \\
\hline HS $1328+3424^{*}$ & 3.7 & 8.7 & 12.0 & 21.2 & 22.32 & 17.11 & 17.02 & 16.07 & 0.94 & 1.06 & 0.48 & 44 & 0.36 & 2.39 \\
\hline HS $1329+3703$ & 3.4 & 7.1 & 9.6 & 21.2 & 23.04 & 17.75 & 17.64 & 16.65 & 0.94 & $0.92-:$ & 0.73 & 91 & 0.37 & 2.86 \\
\hline HS $1330+3651$ & 3.5 & 8.4 & 11.3 & 21.2 & 22.33 & 17.09 & 17.00 & 16.36 & 0.62 & $0.57-$ & 0.65 & 104 & 0.38 & 2.49 \\
\hline HS $1331+3906$ & 4.7 & 9.9 & 13.3 & 21.1 & 22.58 & 16.78 & 16.69 & & & & 0.36 & 155 & 0.33 & 2.43 \\
\hline HS $1332+3426^{*}$ & 2.0 & 4.2 & 6.1 & 21.7 & 22.55 & 18.74 & 18.60 & & & & & & 0.34 & 2.49 \\
\hline HS $1333+3149$ & 3.2 & 9.6 & 14.8 & 20.0 & 21.63 & 16.50 & 16.39 & & & & 0.77 & 104 & 0.58 & 3.04 \\
\hline HS $1333+3717$ & 1.0 & 2.9 & 4.5 & 20.1 & 21.2: & 18.8: & 18.7: & & & & 1. & & 0.52 & 2.59 \\
\hline HS $1334+3957$ & 3.2 & 7.6 & 10.7 & 20.4 & 22.61 & 17.47 & 17.35 & & & & 0.40 & 17 & 0.43 & 3.00 \\
\hline HS $1336+3114$ & 4.5 & 11.6 & 15.8 & 20.9 & 21.94 & 16.15 & 16.08 & 15.08 & 0.94 & $1.11+:$ & 0.79 & 160 & 0.42 & 2.46 \\
\hline HS $1336+3650$ & 2.0 & 5.5 & 7.8 & 20.5 & 21.90 & 17.80 & 17.72 & 16.78 & 0.91 & $1.03+:$ & 0.69 & 63 & 0.49 & 2.77 \\
\hline HS $1337+3941$ & 4.7 & 8.4 & 12.9 & 22.2 & 23.4: & 17.7: & 17.4: & 16.7: & 0.93 & 0.97 & 0.37 & 69 & 0.30 & 2.57 \\
\hline HS $1340+3207$ & 2.2 & 6.0 & 8.4 & 20.6 & 21.69 & 17.57 & 17.48 & & & & 0.65 & 114 & 0.49 & 2.55 \\
\hline HS $1340+3307$ & 1.9 & 5.6 & 8.5 & 20.3 & 21.41 & 17.48 & 17.40 & & & & 0.72 & 30 & 0.53 & 2.61 \\
\hline HS $1341+3117^{*}$ & 1.8 & 5.0 & 7.2 & 20.7 & 22.13 & 18.30 & 18.21 & 17.23 & 0.93 & 0.96 & 0.76 & 150 & 0.54 & 2.97 \\
\hline HS $1341+3409$ & 1.8 & 5.0 & 7.3 & 20.5 & 21.83 & 17.94 & 17.85 & & & & 0.86 & 21 & 0.50 & 2.84 \\
\hline HS $1342+3354^{*}$ & 1.7 & 3.8 & 6.3 & 21.3 & 22.70 & 19.20 & 19.05 & 18.06 & 0.84 & $1.3:+$ & 0.76 & 80 & 0.50 & 2.91 \\
\hline HS $1345+3052$ & 4.6 & 8.9 & 11.7 & 22.5 & 24.01 & 18.23 & 18.08 & 16.95 & 1.12 & $0.95-:$ & 0.81 & 139 & 0.32 & 2.65 \\
\hline
\end{tabular}


Table 4. continued

\begin{tabular}{|c|c|c|c|c|c|c|c|c|c|c|c|c|c|c|}
\hline (1) & $(2)$ & $(3)$ & $(4)$ & (5) & $(6)$ & (7) & $(8)$ & (9) & $(10)$ & $(11)$ & $(12)$ & $(13)$ & $(14)$ & $(15)$ \\
\hline HS $1347+3811$ & 3.0 & 6.5 & 10.0 & 20.8 & 23.03 & 18.03 & 17.83 & 17.11 & 0.64 & $0.97+$ & 0.51 & 84 & 0.44 & 3.42 \\
\hline HS $1349+3942^{*}$ & 3.3 & 9.5 & 14.8 & 20.2 & 22.15 & 16.78 & 16.63 & 15.62 & 0.95 & $1.05+$ & 0.75 & 30 & 0.55 & 3.46 \\
\hline HS $1354+3634$ & 2.2 & 7.4 & 12.3 & 19.7 & 21.71 & 17.20 & 17.06 & 15.94 & 1.00 & $1.35+$ & 0.85 & 66 & 0.62 & 3.67 \\
\hline HS $1354+3635$ & 2.9 & 8.1 & 10.8 & 19.9 & 21.22 & 16.44 & 16.40 & 15.49 & 0.90 & $0.90-:$ & 0.51 & 146 & 0.46 & 2.43 \\
\hline HS $1400+3927$ & 2.5 & 7.4 & 10.9 & 20.7 & 22.80 & 17.96 & 17.82 & 16.58 & 1.22 & $1.43+:$ & 0.67 & 177 & 0.54 & 3.65 \\
\hline HS $1402+3650$ & 2.8 & 8.2 & 11.5 & 19.9 & 21.87 & 17.01 & 16.92 & 16.20 & 0.70 & $0.85+$ & 0.33 & 18 & 0.53 & 3.08 \\
\hline HS $1402+3657$ & 2.3 & 10.4 & 15.2 & 19.4 & 21.20 & 16.55 & 16.48 & 15.32 & 1.11 & $1.24+$ & 0.50 & 91 & 0.69 & 3.79 \\
\hline HS $1404+4015$ & 3.9 & 6.1 & 8.6 & 22.2 & 23.9: & 18.7: & 18.5: & & & & 0.84 & 83 & 0.24 & 2.39 \\
\hline HS $1410+3627$ & 2.8 & 6.1 & 7.9 & 21.4 & 22.22 & 17.62 & 17.56 & 16.84 & 0.67 & $0.80+:$ & 0.50 & 150 & 0.30 & 2.18 \\
\hline HS $1413+4402$ & 2.0 & 5.8 & 7.9 & 20.4 & 21.71 & 17.64 & 17.57 & 16.34 & 1.20 & $1.02-$ & 0.73 & 53 & 0.50 & 2.83 \\
\hline HS $1415+4203$ & 2.4 & 4.9 & 7.1 & 21.7 & 22.60 & 18.40 & 18.29 & 17.15 & 1.12 & $1.14-$ & 0.45 & 162 & 0.31 & 2.30 \\
\hline HS $1416+3554$ & 5.7 & 13.2 & 17.8 & 20.8 & 22.79 & 16.30 & 16.20 & 15.47 & 0.80 & 0.75 & 0.33 & 70 & 0.40 & 2.95 \\
\hline HS $1420+3437$ & 1.2 & 3.8 & 5.6 & 20.2 & 21.22 & 18.32 & 18.24 & & & & 0.90 & 125 & 0.57 & 2.63 \\
\hline HS $1422+3325$ & 1.3 & 3.7 & 5.2 & 20.4 & 21.50 & 18.46 & 18.39 & & & & 0.83 & 41 & 0.49 & 2.60 \\
\hline HS $1422+3339$ & 3.1 & 8.7 & 12.9 & 20.3 & 22.41 & 17.13 & 17.00 & & & & 0.68 & 106 & 0.54 & 3.77 \\
\hline HS $1424+3836$ & 1.6 & 3.5 & 5.8 & 21.2 & 22.19 & 18.82 & 18.68 & 18.69 & $-0.15:$ & $0.2:$ & & & 0.40 & 2.53 \\
\hline HS $1425+3835$ & 2.5 & 6.8 & 9.3 & 20.3 & 21.54 & 17.04 & 16.98 & 16.13 & 0.83 & 0.87 & 0.78 & 167 & 0.47 & 2.58 \\
\hline HS $1429+3154$ & 3.1 & 9.0 & 14.7 & 20.3 & 21.86 & 16.82 & 16.68 & & & & 0.54 & 44 & 0.58 & 3.26 \\
\hline HS $1429+3451$ & 1.8 & 5.8 & 8.4 & 19.8 & 20.93 & 17.28 & 17.20 & & & & 0.64 & 75 & 0.59 & 2.63 \\
\hline HS $1429+4511^{*}$ & 1.5 & 4.2 & 6.3 & 20.4 & 21.80 & 18.30 & 18.22 & 17.07 & 1.12 & 1.15 & 0.89 & 112 & 0.49 & 2.49 \\
\hline HS $1435+4523$ & 2.7 & 5.5 & 7.7 & 21.4 & 22.96 & 18.39 & 18.26 & 17.22 & 0.91 & $0.87-$ & 0.63 & 29 & 0.34 & 2.60 \\
\hline HS $1438+3147$ & 1.6 & 4.2 & 5.7 & 20.7 & 22.11 & 18.46 & 18.38 & 17.66 & 0.65 & $0.8:$ & 0.54 & 154 & 0.43 & 2.64 \\
\hline HS $1440+3120$ & 1.3 & 3.0 & 3.7 & 21.6 & 22.21 & 19.28 & 19.23 & & & & 1. & & 0.33 & 2.20 \\
\hline HS $1440+3805$ & 4.6 & 10.2 & 13.3 & 21.0 & 22.75 & 16.87 & 16.78 & 15.90 & 0.9: & $0.95-:$ & 0.67 & 179 & 0.37 & 2.78 \\
\hline HS $1440+4302$ & 2.3 & 6.1 & 10.1 & 20.5 & 22.36 & 17.89 & 17.70 & 16.89 & 0.80 & $1.05+$ & 0.57 & 29 & 0.55 & 3.48 \\
\hline HS $1442+4250$ & 8.3 & 17.5 & 23.3 & 19.9 & 22.77 & 15.73 & 15.64 & 15.20 & 0.42 & $0.57+:$ & 0.24 & 64 & 0.35 & 2.65 \\
\hline HS $1442+4332$ & 2.0 & 5.2 & 7.2 & 20.6 & 21.44 & 17.62 & 17.55 & & & & 0.88 & 78 & 0.45 & 2.41 \\
\hline HS $1444+3114$ & 2.5 & 9.6 & 14.7 & 19.7 & 21.13 & 16.47 & 16.38 & 15.64 & 0.77 & 0.81 & 0.80 & 30 & 0.67 & 3.31 \\
\hline HS $1450+3844$ & 2.0 & 5.8 & 8.7 & 20.2 & 21.57 & 17.49 & 17.39 & 16.42 & 0.86 & $1.37+$ & 0.84 & 150 & 0.54 & 2.82 \\
\hline HS $1502+4152$ & 4.2 & 8.0 & 10.9 & 22.0 & 23.11 & 17.64 & 17.52 & 16.86 & 0.68 & $0.55-:$ & 0.62 & 159 & 0.28 & 2.35 \\
\hline HS $1504+3922$ & 3.4 & 6.7 & 9.4 & 21.7 & 23.11 & 18.01 & 17.83 & 16.84 & 0.96 & 0.99 & 0.33 & 115 & 0.34 & 2.67 \\
\hline HS $1505+3944$ & 4.3 & 8.3 & 11.9 & 21.6 & 23.17 & 17.58 & 17.41 & 16.08 & 1.31 & 1.37 & 0.77 & 8 & 0.34 & 2.68 \\
\hline HS $1507+3743^{*}$ & 1.6 & 4.4 & 6.4 & 20.0: & 21.64 & 18.13 & 18.01 & 17.78 & $0.2:$ & $0.45:+:$ & 0.77 & 122 & 0.62 & 2.86 \\
\hline HS $1519+4337$ & 4.9 & 7.0 & 11.7 & 22.4 & 24.34 & 18.43 & 18.01 & & & & 0.76 & 153 & 0.33 & 3.27 \\
\hline HS $1522+4214$ & 3.2 & 9.6 & 13.8 & 20.3 & 22.15 & 16.89 & 16.78 & 15.58 & 1.18 & $1.19+:$ & 0.76 & 64 & 0.55 & 3.50 \\
\hline HS $1524+4205$ & 2.7 & 6.7 & 9.6 & 20.8 & 22.43 & 17.67 & 17.55 & 16.60 & 1.03 & $0.80-$ & 0.52 & 62 & 0.45 & 2.93 \\
\hline HS $1526+4045^{*}$ & 4.7 & 9.5 & 14.0 & 21.1 & 23.10 & 17.21 & 17.01 & 16.00 & 0.99 & 0.98 & 0.36 & 16 & 0.37 & 2.97 \\
\hline HS $1529+4512^{*}$ & 2.9 & 6.0 & 10.3 & 21.1 & 22.90 & 18.06 & 17.85 & 16.47 & 1.29 & $1.49+$ & 0.60 & 135 & 0.40 & 3.23 \\
\hline HS $1543+4525$ & 2.7 & 6.7 & 9.0 & 20.6 & 21.44 & 16.90 & 16.86 & 16.26 & 0.50 & $0.64+:$ & 0.88 & 102 & 0.40 & 2.29 \\
\hline HS $1544+4736$ & 1.7 & 5.0 & 6.8 & 20.2 & 21.13 & 17.55 & 17.49 & 17.18 & $0.3:$ & $0.0:$ & 0.85 & 133 & 0.50 & 2.43 \\
\hline HS $1546+4755$ & 1.6 & 4.1 & 5.1 & 20.8 & 21.69 & 18.26 & 18.22 & 17.77 & $0.2:$ & $0.2:$ & 0.76 & 115 & 0.41 & 2.39 \\
\hline HS $1548+4745$ & 1.4 & 3.5 & 4.8 & 20.9 & 21.82 & 18.72 & 18.63 & 17.54 & 1.05 & 1.03 & 0.86 & 31 & 0.41 & 2.39 \\
\hline HS $1549+4630$ & 1.3 & 3.7 & 4.9 & 20.4 & 21.48 & 18.55 & 18.49 & 17.70 & 0.69 & 0.77 & 0.75 & 45 & 0.49 & 2.68 \\
\hline HS $1609+4827$ & 3.9 & 12.9 & 17.9 & 19.5 & 21.21 & 15.51 & 15.47 & & & & 0.90 & 9 & 0.57 & 3.04 \\
\hline HS $1610+4539$ & 1.9 & 5.9 & 7.8 & 20.1 & 21.42 & 17.36 & 17.31 & & & & 1. & & 0.52 & 2.73 \\
\hline HS $1614+4709$ & 2.5 & 8.2 & 11.8 & 20.1 & 21.70 & 17.02 & 16.92 & & & & 0.71 & 20 & 0.60 & 3.16 \\
\hline HS $1626+5153$ & 5.2 & 16.4 & 25.5 & 19.0 & 22.77 & 15.91 & 15.71 & 14.20 & 1.49 & 1.55 & 0.82 & 102 & 0.60 & 6.76 \\
\hline HS $1627+5239$ & 2.2 & 6.2 & 8.8 & 20.5 & 22.03 & 17.60 & 17.50 & 16.33 & 1.15 & 1.07 & 0.63 & 179 & 0.50 & 2.97 \\
\hline HS $1633+4703$ & 2.5 & 8.2 & 13.0 & 20.0 & 21.89 & 17.05 & 16.92 & & & & 0.56 & 176 & 0.60 & 3.59 \\
\hline HS $1634+5218$ & 3.5 & 12.5 & 19.6 & 19.5 & 21.38 & 15.90 & 15.77 & 14.67 & 1.07 & $1.22+$ & 0.74 & 25 & 0.65 & 3.79 \\
\hline HS $1640+5136$ & 2.8 & 9.5 & 12.6 & 19.6 & 20.76 & 15.97 & 15.93 & 14.90 & 0.84 & 1.15 & 0.76 & 134 & 0.59 & 2.64 \\
\hline HS $1641+5053$ & 3.7 & 9.8 & 12.8 & 20.5 & 21.85 & 16.39 & 16.34 & 15.35 & 1.00 & 1.04 & 0.33 & 175 & 0.43 & 2.56 \\
\hline HS $1645+5155$ & 5.7 & 11.7 & 15.5 & 21.0 & 22.53 & 16.36 & 16.27 & 15.35 & 0.89 & $0.78-$ & 0.30 & 23 & 0.31 & 2.40 \\
\hline HS $1657+5033$ & 3.3 & 12.1 & 19.3 & 19.4 & 21.29 & 15.89 & 15.79 & 14.66 & 1.00 & 1.15 & 0.55 & 95 & 0.66 & 3.62 \\
\hline HS $1657+5735$ & 1.9 & 7.8 & 11.7 & 18.8 & 20.06 & 16.02 & 15.98 & 15.11 & 0.75 & $0.95:$ & 0.68 & 109 & 0.69 & 2.68 \\
\hline HS $1723+5631$ & 2.5 & 7.0 & 10.0 & 19.3 & 21.14 & 16.78 & 16.74 & 15.88 & 0.76 & $1.1+:$ & 0.60 & 67 & 0.54 & 2.54 \\
\hline HS $1728+5655$ & 1.2 & 4.6 & 6.4 & 19.4 & 21.19 & 17.95 & 17.89 & 17.06 & 0.75 & 1.2 & 0.63 & 103 & 0.64 & 3.51 \\
\hline HS $1734+5704$ & 1.4 & 5.5 & 7.3 & 19.4 & 20.70 & 17.45 & 17.40 & 16.84 & 0.57 & 1.0 & 0.67 & 47 & 0.66 & 2.90 \\
\hline Residuals & 0.1 & 0.2 & 0.3 & 0.2 & 0.05 & 0.04 & 0.05 & & & & 0.02 & 4 & 0.02 & 0.07 \\
\hline
\end{tabular}


Table 5. Observed model-free photometric data in $R$

\begin{tabular}{lccccccccccc}
\hline Galaxy & $\begin{array}{c}r_{\text {eff }} \\
{\left[{ }^{\prime \prime}\right]}\end{array}$ & $\begin{array}{c}r_{24} \\
{\left[{ }^{\prime \prime}\right]}\end{array}$ & $\begin{array}{c}r_{25.5} \\
{\left[{ }^{\prime \prime}\right]}\end{array}$ & $\begin{array}{c}\mu_{0}^{R} \\
{\left[\frac{\mathrm{mag}}{\square^{\prime \prime}}\right]}\end{array}$ & $\begin{array}{c}\mu_{\mathrm{eff}}^{R} \\
{\left[\frac{\mathrm{mang}}{\square^{\prime \prime}}\right]}\end{array}$ & $\begin{array}{c}R_{24} \\
{[\mathrm{mag}]}\end{array}$ & $\begin{array}{c}R_{\mathrm{T}} \\
{[\mathrm{mag}]}\end{array}$ & $b / a$ & $\begin{array}{c}\mathrm{PA} \\
{\left[{ }^{\circ}\right]}\end{array}$ & $c_{\text {in }}$ & $c_{31}$ \\
\hline (1) & $(2)$ & $(3)$ & $(4)$ & $(5)$ & $(6)$ & $(7)$ & $(8)$ & $(9)$ & $(10)$ & $(11)$ & $(12)$ \\
\hline HS 0016+1449 & 1.5 & 7.0 & 9.3 & 17.4 & 18.71 & 15.14 & 15.12 & 0.63 & 32 & 0.69 & 2.97 \\
HS 0029+1443 & 2.1 & 5.8 & 7.6 & 19.6 & 20.72 & 16.57 & 16.52 & 0.75 & 84 & 0.48 & 2.61 \\
HS 0029+1748 & 3.5 & 8.2 & 11.7 & 19.9 & 21.97 & 16.57 & 16.43 & 0.48 & 134 & 0.45 & 3.32 \\
HS 0040+0952 & 2.2 & 5.8 & 8.5 & 19.8 & 20.97 & 16.74 & 16.64 & 0.61 & 94 & 0.46 & 2.70 \\
HS 0043+0531 & 2.3 & 5.0 & 6.9 & 20.7 & 21.94 & 17.64 & 17.52 & 0.80 & 173 & 0.37 & 2.67 \\
HS 0051+0927 & 1.8 & 4.4 & 5.7 & 20.3 & 21.08 & 17.50 & 17.44 & & & 0.38 & 2.41 \\
HS 0052+2119 & 2.3 & 5.0 & 6.9 & 20.6 & 21.52 & 17.33 & 17.24 & 0.75 & 0 & 0.35 & 2.41 \\
HS 0058+1847 & 1.7 & 4.5 & 5.8 & 19.9 & 20.81 & 17.22 & 17.17 & & & 0.43 & 2.45 \\
HS 0110+2149 & 6.9 & 18.1 & 23.0 & 19.3 & 21.90 & 14.78 & 14.69 & 0.79 & 15 & 0.47 & 3.76 \\
HS 0111+2115 & 4.1 & 8.9 & 11.7 & 20.3 & 21.47 & 15.93 & 15.87 & 0.85 & 132 & 0.33 & 2.38 \\
HS 0113+1750 & 1.5 & 3.6 & 5.2 & 20.5 & 21.41 & 18.09 & 17.96 & & & 0.39 & 2.50 \\
HS 0123+1624 & 2.9 & 7.2 & 8.5 & 20.2 & 21.57 & 16.70 & 16.64 & 0.50 & 7 & 0.41 & 2.59 \\
HS 0133+1341 & 2.1 & 6.2 & 8.7 & 19.6 & 20.81 & 16.63 & 16.54 & 0.87 & 44 & 0.53 & 2.71 \\
HS 0143+2400 & 1.7 & 4.3 & 6.1 & 20.2 & 21.53 & 17.76 & 17.64 & 0.57 & 37 & 0.46 & 2.87 \\
HS 0148+1700 & 2.3 & 6.3 & 8.7 & 19.5 & 20.41 & 16.15 & 16.10 & 0.67 & 63 & 0.45 & 2.40 \\
HS 0148+2123 & 2.8 & 7.6 & 11.9 & 19.5 & 20.99 & 16.18 & 16.07 & 0.78 & 24 & 0.49 & 2.78
\end{tabular}

Table 6. Reduced photometric data and exponential model parameters in $B$ and morphological classes

\begin{tabular}{|c|c|c|c|c|c|c|c|c|c|c|c|c|c|}
\hline \multirow[t]{2}{*}{ Galaxy } & \multirow{2}{*}{$\begin{array}{r}D \\
{[\mathrm{Mpc}]}\end{array}$} & \multirow{2}{*}{$\begin{array}{c}M_{B} \\
{[\mathrm{mag}]}\end{array}$} & \multirow{2}{*}{$\begin{array}{r}r_{\text {eff }} \\
{[\mathrm{kpc}]}\end{array}$} & \multirow{2}{*}{$\begin{array}{r}r_{25} \\
{[\mathrm{kpc}]}\end{array}$} & \multirow{2}{*}{$\begin{array}{r}r_{\mathrm{H} \text { II }} \\
{[\mathrm{kpc}]}\end{array}$} & \multicolumn{2}{|c|}{$\alpha^{-1}$} & \multirow{2}{*}{$\begin{array}{l}\mu_{0}^{\exp } \\
{\left[\frac{\mathrm{mag}}{\square^{\prime \prime}}\right]}\end{array}$} & \multirow{2}{*}{$\begin{array}{l}\mu_{0, \mathrm{c}}^{\exp } \\
{\left[\frac{\mathrm{mag}}{\square^{\prime \prime}}\right]}\end{array}$} & \multirow{2}{*}{$\begin{array}{l}\text { profile } \\
\text { type }\end{array}$} & \multicolumn{3}{|c|}{ morph. classes } \\
\hline & & & & & & {$\left[{ }^{\prime \prime}\right]$} & {$[\mathrm{kpc}]$} & & & & SMB89 & LTh85 & TMT97 \\
\hline (1) & $(2)$ & $(3)$ & $(4)$ & $(5)$ & $(6)$ & (7) & $(8)$ & (9) & $(10)$ & $(11)$ & $(12)$ & $(13)$ & $(14)$ \\
\hline HS $0731+6348$ & 1380.4 & -22.55 & 8.7 & 23.0 & 3.7 & 1.0: & 7.0: & $21.2:$ & 21.3: & 4 st/d: & Sy 1 [lit] & iI: & 12 \\
\hline HS $0732+6503$ & 88.5 & -18.02 & 1.4 & 3.5 & 0.9 & 2.0: & 0.9: & 20.6: & 20.7: & $2 \mathrm{n} / \mathrm{bd}:$ & DANS & $\mathrm{n}: \mathrm{E}$ & 12 \\
\hline HS $0749+5649$ & 77.1 & -16.93 & 0.7 & 2.0 & 0.4 & & & & & $4 \mathrm{st}$ & DHIIH: & $\mathrm{nE} / \mathrm{i} 0$ & 12 \\
\hline HS $0752+5603$ & 111.1 & -18.85 & 1.8 & 5.7 & 1.1 & 3.9 & 2.1 & 21.98 & 21.98 & $2 \mathrm{bd}$ & DANS/HIIH & nI: & 12 \\
\hline HS $0757+6441$ & 294.8 & -19.89 & 3.0 & 8.6 & 2.2 : & 1.5: & $2.2:$ & 21.1: & $21.5:$ & 2 bd: & $\mathrm{SBN} / \mathrm{HIIH}$ & $\mathrm{nI}, \mathrm{M}$ & 11 \\
\hline HS $0805+5742$ & 109.5 & -17.70 & 1.0 & 2.8 & 0.6 & 1.0: & $0.5:$ & 19.6: & 19.7: & 4 st/d: & DANS & $\mathrm{nE}$ & 12 \\
\hline HS $0831+6215$ & 76.2 & -16.92 & 0.6 & 1.8 & 0.4 & & & & & $4 \mathrm{st}$ & DHIIH & $\mathrm{nE} / \mathrm{i} 0$ & 12 \\
\hline HS $0915+5540$ & 198.6 & -19.30 & 1.9 & 5.7 & 1.0 & 1.8 & 1.8 & 21.30 & 21.57 & $2 \mathrm{bd}$ & DANS & $\mathrm{iE}$ & 12 \\
\hline HS $1222+3741$ & 164.0 & -17.67 & 1.0: & 2.5: & 0.5 & & & & & $4 \mathrm{st}$ & $\mathrm{BCD}$ & $\mathrm{iE}$ & 12 \\
\hline HS $1223+3938$ & 144.5 & -18.73 & 2.2 & 5.5 & $1.2:$ & 1.9 & 1.3 & 20.20 & 20.70 & $1 \mathrm{nd}$ & HIIH & S.. & 11 \\
\hline HS $1226+3620$ & 272.4 & -18.69 & 3.7 & 7.0 & 3.3: & 1.6 & 2.2 & 21.46 & 21.72 & $1 \mathrm{~d}$ & HIIH & iI, C: & 31 \\
\hline HS $1226+3719 *$ & 44.5 & -14.06 & 0.4 & 0.7 & 0.2 & & & & & 4 st & [nonELG] & iI & 12 \\
\hline HS $1232+3609$ & 1012.0 & -20.96 & 9.1 & 20.9 & 3.2 & 1.8 & 8.8 & 22.43 & 22.91 & 2 nd:c: & SBN & S.. & 11 \\
\hline HS $1232+3612$ & 170.4 & -19.77 & 3.0 & 7.8 & & 5.2 & 4.3 & 23.22 & 23.61 & $2 \mathrm{~b}: \mathrm{d}:$ & IP & iI,M & 21 \\
\hline HS $1232+3846$ & 211.7 & -19.70 & 6.7 & 11.9 & 2.0 & 4.9 & 5.0 & 22.37 & 23.37 & $2 \mathrm{bd}$ & $\mathrm{SBN}$ & S.. & 31 \\
\hline HS $1232+3947^{*}$ & 84.6 & -17.16 & 0.9 & 2.4 & 0.7 : & 1.7 & 0.7 & 21.14 & 22.31 & 2 nd & DHIIH & iI,M: & 21 \\
\hline HS $1236+3821 *$ & 29.7 & -17.06 & 1.3 & 2.8 & $0.3:$ & 12.5 & 1.8 & 23.26 & 23.73 & $2 \mathrm{n} / \mathrm{bd}$ & DHIIH & iI,M & 31 \\
\hline HS $1236+3937 *$ & 74.2 & -15.67 & 1.0 & 1.6 & $0.3:$ & 2.7 & 1.0 & 23.00 & 23.99 & 2 nd:d & DHIIH/SS & iI, C & 31 \\
\hline HS $1240+3721^{*}$ & 87.7 & -16.90 & 1.8 & 3.1 & 1.3 & 2.7 & 1.1 & 21.88 & 22.06 & $1 \mathrm{~d}$ & DHIIH & $\mathrm{nE}$ & 12 \\
\hline HS $1240+3755$ & 344.6 & $-21.1:$ & 5.2 & 13.8 & & 1.8 & 3.0 & 19.8: & 20.2 : & $1 \mathrm{~d}$ & IP & $\mathrm{iE}:, \mathrm{M}$ & 21 \\
\hline HS $1242+3551$ & 636.0 & -19.95 & 6.3 & 12.0 & & 1.6 & 4.9 & 20.60 & 20.94 & $1 \mathrm{~d}:$ & $\mathrm{SBN} / \mathrm{GI}$ & nI/S.. & 12 \\
\hline HS $1244+3648$ & 189.3 & -18.82 & 1.2 & 4.1 & 0.7 & & & & & $4 \mathrm{st}$ & DANS & $\mathrm{nE} / \mathrm{i} 0$ & 12 \\
\hline HS $1254+3323$ & 13.2 & -12.45 & 0.1 & 0.2 & & & & & & 4 st & [nonELG] & i0 & 12 \\
\hline HS $1255+3506$ & 62.5 & -16.61 & 0.7 & 1.9 & 0.5 & & & & & $5 \mathrm{cnc}$ & DHIIH: & $\mathrm{nE}$ & 12 \\
\hline HS $1256+3505$ & 137.3 & -18.75 & 1.1 & 4.7 & 0.7 & 2.5 & 1.7 & 21.91 & 22.29 & $2 \mathrm{bd}$ & DANS & $\mathrm{nE}$ & 12 \\
\hline HS $1258+3438$ & 99.7 & -16.43 & 1.2 & 2.3 & & 2.1 & 1.0 & 22.58 & 23.40 & 2 nd & HIIH/DHIIH & iI,C: & 22 \\
\hline HS $1301+3209$ & 95.6 & -17.06 & 1.1 & 2.4 & 1.0 & 1.1: & $0.5:$ & 19.9: & $20.0:$ & $4 \mathrm{~cd}: / \mathrm{st}$ & $\mathrm{BCD}$ & i0: & 12 \\
\hline HS $1301+3312$ & 148.8 & -17.41 & 0.9 & 2.6 & 0.6 & 1.1 & 0.8 & 21.49 & 21.69 & 2 nd & HIIH & iI,M: & 12 \\
\hline HS $1301+3325$ & 98.9 & -16.96 & 0.8 & 2.2 & 0.6 & 1.0: & 0.5 : & 20.1: & $20.2:$ & $4 \mathrm{st}$ & DHIIH & $\mathrm{nE}$ & 12 \\
\hline HS $1302+3046$ & 142.3 & -19.34 & 4.6 & 8.9 & $2.2:$ & & & & & $3 \mathrm{cnv}$ & SBN: & S.. & 12 \\
\hline HS $1304+3529$ & 66.6 & -17.38 & 1.0 & 2.7 & 0.6 : & 1.8 & 0.6 & 20.02 & 20.29 & $1 \mathrm{dh}:$ & HIIH & iI,M: & 21 \\
\hline HS $1306+3320 *$ & 108.9 & -18.32 & 2.0 & 4.9 & 1.0 & & & & & $5 \mathrm{cnc}$ & HIIH & iI,C & 31 \\
\hline HS $1306+3525$ & 66.6 & -17.19 & 1.2 & 3.0 & $0.9:$ & 2.6 & 0.8 & 21.01 & 21.64 & $1 \mathrm{~d}:$ & HIIH & $\mathrm{n}: \mathrm{I}$ & 11 \\
\hline HS $1306+3527$ & 149.0 & -18.55 & 2.0 & 5.4 & 1.0 & 1.9 & 1.4 & 20.80 & 21.10 & $1 \mathrm{~d}$ & DANS & $\mathrm{nE}$ & 12 \\
\hline HS $1308+3044$ & 84.0 & -17.92 & 1.1 & 3.2 & 1.0: & 1.5: & $0.6:$ & 19.6: & $20.5:$ & 2 c:d:h: & DANS & $\mathrm{iE} / \mathrm{S} .$. & 12 \\
\hline HS $1309+3409$ & 314.6 & -20.19 & 3.8 & 10.6 & 2.3 & 2.3 & 3.5 & 21.50 & 21.78 & $2 \mathrm{bd}$ & $\mathrm{SBN}$ & $\mathrm{iE}$ & 12 \\
\hline HS $1311+3628$ & 13.1 & -16.84 & 2.0 & 3.3 & & 18.6 & 1.2 & 21.99 & 22.26 & $3 \mathrm{c}: \mathrm{d}$ & DHIIH/HIIH & iI & 31 \\
\hline HS $1312+3112$ & 82.4 & -14.4 : & 1.6 & & & & & & & $3 \mathrm{cnv}$ & DHIIH & iI,C & 32 \\
\hline HS $1312+3508$ & 14.6 & -16.26 & 0.9 & 2.0 & & 8.4 & 0.6 & 21.35 & 21.80 & $2 \mathrm{nd}$ & DHIIH & i0 & 32 \\
\hline HS $1312+3847$ & 206.8 & -18.77 & 1.8 & 5.2 & 1.4: & 2.0 & 2.0 & 22.21 & 22.78 & 2 nd & HIIH & iI,C: & 21 \\
\hline HS $1314+3320$ & 206.6 & $-16.9:$ & 1.0 & 2.3: & 0.6 : & 1.0: & 1.0: & $22.7:$ & 22.9: & 4 st/nd: & HIIH/DHIIH & iI:/i0 & 12 \\
\hline
\end{tabular}


Table 6. continued

\begin{tabular}{|c|c|c|c|c|c|c|c|c|c|c|c|c|c|}
\hline (1) & $(2)$ & $(3)$ & $(4)$ & $(5)$ & $(6)$ & (7) & $(8)$ & (9) & (10) & (11) & $(12)$ & (13) & $(14)$ \\
\hline HS $1315+3132$ & 126.5 & -16.46 & 0.8 & 1.9 & 0.5 & & & & & $4 \mathrm{st}$ & DHIIH & i0 & 12 \\
\hline HS $1318+3239$ & 174.6 & 17.22 & 1.3 & 3.0 & 1.0: & 1.0: & $0.8:$ & 20.9: & 21.5: & $1 \mathrm{~d}:$ & HIIH & iI,M: & 12 \\
\hline HS $1318+3406$ & 141.4 & 18.65 & 1.9 & 4.5 & & & & & & $3 \mathrm{~cd}:$ & HIIH: & S.. & 32 \\
\hline HS $1319+3224$ & 73.3 & $-15.3:$ & 0.5 & 1.3: & 0.3 & 1.5 & 0.5 & 22.3: & $22.7:$ & 2 nd: & DHIIH & $\mathrm{iE}$ & 12 \\
\hline HS $1325+3225$ & 202.2 & -17.9 : & 1.5 & 3.7: & & 0.9: & 0.9: & 20.4: & 20.3: & $1 \mathrm{~d}$ & DANS & $\mathrm{nE} / \mathrm{i} 0$ & 12 \\
\hline HS $1325+3255^{*}$ & 105.8 & $-15.9:$ & $0.7:$ & 1.5: & 0.4 & & & & & $4 \mathrm{nd}: / \mathrm{st}$ & DHIIH/SS & $\mathrm{iE}^{\prime}$ & 12 \\
\hline HS $1327+3126$ & 227.8 & -18.07 & 1.3 & 3.3 & 0.7 & & & & & 4 st & DHIIH & i0 & 12 \\
\hline HS $1327+3412$ & 1004.7 & $-21.1:$ & 5.7 & 14.8: & 3.4 & 1.3: & $6.2:$ & 22.4: & 22.4: & 4 st/nd: & SBN & $\mathrm{nE}:$ & 12 \\
\hline HS $1327+3749$ & 218.1 & $-18.1:$ & 2.1 & 4.7: & 1.6: & 1.3 & 1.3 & 21.13 & 21.76 & $1 \mathrm{~d}$ & DANS & $\mathrm{nE}:$ & 12 \\
\hline HS $1328+3424^{*}$ & 91.5 & -17.79 & 1.7 & 3.8 & & 2.3 & 1.0 & 20.80 & 21.60 & $3 \mathrm{~cd}:$ & HIIH & $\mathrm{iE}, \mathrm{C}:$ & 32 \\
\hline $29+3703$ & 223.7 & 19.11 & 3.7 & 7.7 & 2.1: & 2.0 & 2.1 & 21.05 & 21.39 & $1 \mathrm{~d}:$ & HIIH & iI & 11 \\
\hline $30+3651$ & 67.7 & -17.15 & 1.2 & 2.7 & & 2.1 & 0.7 & 20.55 & 21.02 & $1 \mathrm{~d}:$ & DHIIH/HIIH & iI,M: & 31 \\
\hline $31+3906$ & 258.2 & -20.37 & 5.8 & 12.3 & 3.0: & 2.3: & 2.8: & $20.2:$ & 21.3: & 3 c:d: & SBN & $\mathrm{S}$ & 31 \\
\hline $2+3426$ & 88.8 & -16.14 & 0.8 & 1.8 & 0.6 & & & & & $4 \mathrm{st}$ & DHIIH & $\mathrm{nE} / \mathrm{i} 0$ & 12 \\
\hline HS $1333+3149$ & 99.8 & -18.61 & 1.5 & 4.6 & 1.1: & 3.8 & 1.8 & 22.19 & 22.47 & $2 \mathrm{bd}$ & HIIH & $\mathrm{i}: \mathrm{E}$ & 12 \\
\hline $3+3717$ & 228.6 & $-18.1:$ & 1.1 & 3.2 & 0.6 & 1.4: & 1.6: & 23.0: & 23.0: & $4 \mathrm{st}$ & ANS & i0 & 12 \\
\hline HS 1 & 34.3 & -15.32 & 0.5 & 1.3 & $0.3:$ & 2.1 & 0.3 & 20.95 & 21.94 & $1 \mathrm{n}: \mathrm{d}$ & ОНIIH & iI & 21 \\
\hline HS 1 & 3.8 & -17.94 & 1.4 & 3.6 & 1.2: & 2.5 & 0.8 & 19.96 & 20.22 & $3 \mathrm{c}: \mathrm{d}$ & HIIH & $\mathrm{iE}$ & 22 \\
\hline HS 1 & 81.7 & -16.84 & 0.8 & 2.2 & 0.6 : & 1.5 & 0.6 & 21.03 & 21.43 & $2 \mathrm{nd}$ & HIIH & $\mathrm{nE}$ & 12 \\
\hline HS 1 & 11.1 & -12.80 & 0.3 & 0.4 & & 2.7 & 0.1 & 21.48 & 22.56 & $1 \mathrm{c}$ & S/IM & $\mathrm{iE}$ & 32 \\
\hline HS 1 & 146.7 & -18.35 & 1.5 & 4.3 & 1.2 & 1.7 & 1.2 & 21.12 & 1.59 & $2 \mathrm{n} / \mathrm{bd}$ & NS & $\mathrm{nE}:$ & 12 \\
\hline HS 1 & 64.0 & -16 . & 0.6 & 1.7 & 0.5 & & & & & $4 \mathrm{st}$ & DHIIH & $\mathrm{i}: \mathrm{E}$ & 12 \\
\hline HS 1 & 117.9 & 17. & 1.0 & 2.9 & 0.7 & & & & & & DANS/DHIIH & $n E:$ & 12 \\
\hline HS 13 & 69.2 & -16 . & 0.6 & 1.7 & 0.4 & & & & & & DHIIH & $\mathrm{nE}$ & 12 \\
\hline HS 1 & 22.4 & -12 . & 0.2 & 0.4 & 0.1 & 1.8: & 0.2 : & 22.8: & 23.1: & & & $\mathrm{nE}:$ & 12 \\
\hline HS $1:$ & 307.9 & -19 & 6.9 & 13.3 & 4.5: & 2.9 & 4.3 & 22.30 & 22.52 & $1 \mathrm{dc}$ & IIH & $\mathrm{nI}$ & 21 \\
\hline HS $1:$ & 2.3 & -15 & 0.6 & 1.3 & 1.0: & & & & & & IIH & iI:, C & 22 \\
\hline HS $1:$ & 22.8 & -15.16 & 0.4 & 1.0 & 0.2 & 4.6: & 0.5 : & 22.8: & 23.1: & & IIH & & 12 \\
\hline HS 1 & 67.9 & -17.10 & 0.7 & 2.4 & 0.4 & & & & & $5 \mathrm{cnc}$ & DANS & $\mathrm{nE}$ & 12 \\
\hline HS 1 & 9.5 & -17 . & 1.0 & 2.7 & 1.1 & 1.5: & $0.5:$ & 19.0: & 19.8: & $3 \mathrm{c}$ & & $\mathrm{iE}, \mathrm{C}$ & 22 \\
\hline HS 1 & & -13 & 0. & 0.7 & 0.2 & 2.4 & 0.2 & 22.23 & 22.66 & & & & 12 \\
\hline $\mathrm{S} 1$ & 140.0 & -18. & 1.9 & 5.5 & 1.1: & 2.3 & 1.6 & 21.12 & 22.32 & & $\mathrm{IH}$ & iI,M: & 31 \\
\hline S 1 & & 16 & 0.6 & 2.5 & 0.3 & 3.1 & 0.7 & 21.76 & 22.51 & 21 & IIIH & M: & 11 \\
\hline HS 1 & 8.9 & $-20.5:$ & 11.6 & 18.0 & 9.9: & 2.0 & 5.8 & 21.7: & 21.9: & $1 \mathrm{~d}$ & & $\mathrm{nE}$ : & 12 \\
\hline HS 1 & 6.4 & -18.11 & 1.8 & 4.0 & & & & & & $3 \mathrm{~cd}:$ & & iI,C: & 32 \\
\hline S 1 & 30.8 & -19 . & 2.7 & 8.0 & 1.7 & 1.2 & 1.7 & 20.04 & 20.38 & & & & 12 \\
\hline HS 1 & 74.7 & -18 & 3.2 & 6.6 & 3.0: & 1.3 & 1.7 & 0.70 & 1.57 & $3 \mathrm{~cd}$ & НIIH & $\mathrm{nE}:$ & 12 \\
\hline HS 1 & 2.4 & -16 . & 1.2 & 2.7 & 0.7 : & 3.4 & 0.7 & 20.85 & 22.05 & $1 \mathrm{c}$ & $\mathrm{IIH} / \mathrm{MI}$ & iI/S. & 32 \\
\hline HS 1 & & -16 & 0.6 & 1.8 & 0.3 & 1.3: & 0.6 : & 21.7: & 21.8: & & & & 12 \\
\hline HS 1 & 137.6 & -17 & 0.9 & 2.5 & 0.5 & 1.1: & $0.7:$ & 21.3: & 21.5: & $4 \mathrm{st} / \mathrm{d}:$ & DANS/DHIIH & $\mathrm{nE}$ & 12 \\
\hline HS 1 & & -16 & 0 . & 2.0 & 0.4 & 2.9 & 0.7 & 21.75 & 22.17 & & D & & 12 \\
\hline HS 1 & & 16 & 0 & 1.5 & 0.6 & & & & & & & $\mathrm{nI} / \mathrm{i} 0$ & 12 \\
\hline S 1 & & 17 & 1. & 3.0 & 0.8 & 1.7 & 0.8 & 20.6 & 20.92 & & $\mathrm{~S} / \mathrm{D}$ & & 12 \\
\hline S 1 & & -16 & 0. & 2.1 & 0.6 : & 4.2 & 1.0 & 0 & 3.37 & & & $\mathrm{iE}, \mathrm{C}$ & 22 \\
\hline HS 1 & 58.9 & -16 & 0.5 & 1.7 & $0.4:$ & 1.7 & 0.5 & 21.24 & 21.72 & 2 ndh: & & & 12 \\
\hline & & & 1.0 & 2.7 & 0.6 & & & & & & $\mathrm{NS} / \mathrm{D}$ & $\mathrm{nE} /$ & 12 \\
\hline S 1 & 8.6 & -20 . & 6. & 13.5 & 4.8: & 1.6 & 3.9 & 21.21 & 21.69 & & & S.. & 11 \\
\hline HS 1 & & -17 . & 1.4 & 3.6 & 1.0 & 1.0 & 0.9 & 20.26 & 20.93 & $1 \mathrm{~d}$ & & $\mathrm{I}$ & 12 \\
\hline HS 1 & & -17 & 1.4 & & 0.7 & & & & & & IIH/I & i0 & 12 \\
\hline HS 1 & 0.4 & -18 & 2. & 6.5 & 1.8: & 2.7 & 1.7 & 20.88 & & & & & 31 \\
\hline $\mathrm{H}$ & & -15 . & 0. & 1.1 & 0.2 & 2.7 & 0.5 & 2.40 & 23.00 & & $\mathrm{HIIH} / \mathrm{SS}$ & & 12 \\
\hline $\mathrm{H}$ & & -14 & 0 & 1.0 & & 4.2 & 0.2 & 20.53 & 22.07 & & & & 22 \\
\hline & & -20 & 3 & 8.3 & 2.3 & 1.3: & 2.0: & & 20 & 4 & & & 12 \\
\hline & 120.1 & -19 & 1. & 5.6 & 1.0: & 3.5 & 2.0 & & 22.21 & & & & 31 \\
\hline $\mathrm{H}$ & & -16 & 0 & 1.6 & 0.4 & 2.3 & 0.6 & 22.35 & 22.52 & & & & 12 \\
\hline $\mathrm{H}$ & & -16 & 1. & 2.6 & & & & & & & & iI,C: & 32 \\
\hline & & & 2. & 4.0 & 1.3: & 2.0 & 1.2 & & & & & & 12 \\
\hline $\mathrm{H}$ & & -18 & & 6.0 & 2.0 & 2.5 & 1.8 & 21.40 & 21.65 & & & & 12 \\
\hline HS 1 & & -17 . & 1.0 & 2.8 & 0.6 & & & & & $4 \mathrm{st}$ & HIIH/DHIIH & $\mathrm{iI} / \mathrm{i} 0$ & 11 \\
\hline & & -20 . & 12.7 & 18.3 & 5.1: & 3.5 & & & & & & $\mathrm{nE} / \mathrm{S} .$. & 12 \\
\hline HS 1 & & -17 & 1.2 & & 0.7 & 3.1 & 1.2 & 21 & 21. & & & & 12 \\
\hline HS $1:$ & & -17 . & 1.2 & 3.0 & 0.8 & 1.6 & 0.7 & 20.60 & 21.23 & $1 \mathrm{dh}:$ & NS: & $\mathrm{nE}$ & 12 \\
\hline & 117.3 & & 2.7 & & 1.0: & 2.8 & 1.6 & 21.26 & 22.31 & $1 \mathrm{~d}$ & & & 21 \\
\hline HS 1 & & -17 & 1 . & 2.8 & $0.8:$ & 2.9: & 1.4: & 22.8: & 23.3: & & $\mathrm{H} / \mathrm{DH}$ & iI,C & 21 \\
\hline HS 1 & 158.0 & -19 & 2. & 5.2 & 1.7 & & & & & $4 \mathrm{cnv} / \mathrm{st}$ & & & 12 \\
\hline & & & & 2.0 & 1.0 & & & & & & & $\mathrm{iE}$ & 22 \\
\hline HS $1:$ & & -17 . & 1.2 & 3.0 & 0.5 & & & & & & NS/DHIIH & nI:,M: & 12 \\
\hline HS 1 & 282.5 & -18 & 1.9 & $4 .^{\prime} \quad$ & 1.4 & 0.8 & 1.1 & & & & & $\mathrm{nE}$ & 12 \\
\hline & & & 2.4 & 7.0 & 1.4: & $0.7:$ & 1.4: & & 20.0: & & & nI & 11 \\
\hline HS $1609+4827$ & 41.1 & -17.60 & 0.8 & 2.6 & 0.5 : & 2.7: & 0.5 : & 19.7: & 19.8: & $5 \mathrm{cnc} / \mathrm{d}:$ & HIIH/DHIIH & $\mathrm{iE}$ & 12 \\
\hline
\end{tabular}


Table 6. continued

\begin{tabular}{|c|c|c|c|c|c|c|c|c|c|c|c|c|c|}
\hline$(1)$ & $(2)$ & $(3)$ & $(4)$ & $(5)$ & $(6)$ & (7) & $(8)$ & (9) & $(10)$ & $(11)$ & $(12)$ & $(13)$ & (14) \\
\hline HS $1610+4539$ & 81.0 & -17.23 & 0.8 & 2.3 & 0.5 & 1.2 & 0.5 & 19.78 & 19.78 & $1 \mathrm{~d}$ & DANS & $\mathrm{nE} / \mathrm{i} 0$ & 12 \\
\hline HS $1614+4709$ & 13.1 & -13.66 & 0.2 & 0.5 & 0.1 & 2.9 & 0.2 & 21.95 & 22.32 & $2 \mathrm{nd}$ & SS & i:E & 12 \\
\hline HS $1626+5153$ & 221.7 & -21.04 & 5.6 & 17.7 & 1.2 & 6.8 & 7.3 & 22.39 & 22.59 & $2 \mathrm{bd}$ & Sy 1[lit] & $\mathrm{nE} / \mathrm{S}$. . & 12 \\
\hline HS $1627+5239$ & 118.1 & -17.88 & 1.3 & 3.5 & 0.7 & 1.8: & 1.0: & 21.2: & 21.7: & $5 \mathrm{cnc} / \mathrm{d}:$ & HIIH & $\mathrm{iE}$ & 12 \\
\hline HS $1633+4703$ & 37.2 & -15.93 & 0.5 & 1.5 & 0.2 & 3.5 & 0.6 & 22.5 & 23.1 & 2 nd: & DHIIH & $\mathrm{nE}$ & 12 \\
\hline HS $1634+5218$ & 37.7 & -17.14 & 0.6 & 2.3 & $0.4:$ & 5.5: & 1.0: & 22.6: & 22.9: & 2 nd: & $\mathrm{BCD}$ & iI & 12 \\
\hline HS $1640+5136$ & 126.2 & -19.59 & 1.7 & 5.8 & 1.2 & 2.3: & 1.4: & 20.5: & 20.8: & 2 bd: & $\mathrm{SBN} / \mathrm{HIIH}$ & $\mathrm{nI}$ & 11 \\
\hline HS $1641+5053$ & 119.8 & -19.05 & 2.2 & 5.7 & & 2.1 & 1.2 & 19.83 & 21.03 & $3 \mathrm{~cd}:$ & HIIH & $\mathrm{iI}, \mathrm{M}$ & 31 \\
\hline HS $1645+5155$ & 117.4 & -19.11 & 3.3 & 6.7 & 3.1: & 3.3: & 1.9: & $21.2:$ & 22.5: & $3 \mathrm{cnv} / \mathrm{d}:$ & HIIH & iI & 11 \\
\hline HS $1657+5033$ & 43.9 & -17.43 & 0.7 & 2.6 & 0.4 & 5.2 & 1.1 & 22.50 & 23.14 & 2 nd: & HIIH & $\mathrm{nI}$ & 11 \\
\hline HS $1657+5735$ & 205.2 & -20.60 & 1.9 & 7.8 & 1.2: & & & & & $5 \mathrm{cnc}$ & SBN & iI,C: & 11 \\
\hline HS $1723+5631$ & 117.7 & -18.70 & 1.4 & 4.0 & & 2.3 & 1.3 & 21.56 & 22.02 & $2 \mathrm{n} / \mathrm{b}: \mathrm{d}:$ & IP & iI,M & 22 \\
\hline HS $1728+5655$ & 67.3 & -16.38 & 0.4 & 1.5 & 0.3 & 1.4: & $0.5:$ & 21.5: & 21.8: & 2 nd: & DHIIH & $\mathrm{nE}$ & 12 \\
\hline HS $1734+5704$ & 193.4 & -19.15 & 1.3 & 5.2 & 0.5 & 1.5 & 1.4 & 21.17 & 21.48 & 2 nd & HIIH & iI & 11 \\
\hline Residuals & & & & & & 0.15 & & 0.2 & & & & & \\
\hline
\end{tabular}

Table 7. Reduced photometric data and exponential model parameters in $R$ and morphological classes

\begin{tabular}{|c|c|c|c|c|c|c|c|c|c|c|c|c|c|}
\hline \multirow[t]{2}{*}{ Galaxy } & \multirow{2}{*}{$\begin{array}{r}D \\
{[\mathrm{Mpc}]}\end{array}$} & \multirow{2}{*}{$\begin{array}{c}M_{R} \\
{[\mathrm{mag}]}\end{array}$} & \multirow{2}{*}{$\begin{array}{r}r_{\text {eff }} \\
{[\mathrm{kpc}]}\end{array}$} & \multirow{2}{*}{$\begin{array}{r}r_{24} \\
{[\mathrm{kpc}]}\end{array}$} & \multirow{2}{*}{$\begin{array}{r}r_{\mathrm{HII}} \\
{[\mathrm{kpc}]}\end{array}$} & \multicolumn{2}{|c|}{$\alpha^{-1}$} & \multirow{2}{*}{$\begin{array}{c}\mu_{0}^{\exp } \\
{\left[\frac{\mathrm{mag}}{\mathrm{D}^{\prime \prime}}\right]}\end{array}$} & \multirow{2}{*}{$\begin{array}{l}\mu_{0, \mathrm{c}}^{\exp } \\
{\left[\frac{\mathrm{mag}}{\mathrm{D}^{\prime \prime \prime}}\right]}\end{array}$} & \multirow{2}{*}{$\begin{array}{l}\text { profile } \\
\text { type }\end{array}$} & \multicolumn{3}{|c|}{ morph. classes } \\
\hline & & & & & & {$\left[{ }^{\prime \prime}\right]$} & {$[\mathrm{kpc}]$} & & & & SMB89 & Lih85 & ТМТ97 \\
\hline (1) & (2) & (3) & (4) & (5) & (6) & (7) & (8) & (9) & (10) & $(11)$ & $(12)$ & (13) & $(14)$ \\
\hline HS $0016+1449$ & 61.3 & -18.83 & 0.5 & 2.1 & 0.1 & 1.5: & $0.4:$ & 18.7: & 19.2: & $4 \mathrm{st}$ & HIIH/DHIIH & $\mathrm{iE}:$ & 12 \\
\hline HS $0029+1443$ & 72.4 & -17.80 & 0.7 & 2.0 & 0.5 & 1.2 & 0.4 & 18.86 & 19.16 & $1 \mathrm{~d}$ & DHIIH & $\mathrm{nE}$ & 12 \\
\hline HS $0029+1748$ & 31.8 & -16.10 & 0.5 & 1.3 & 1.8 & 0.2 & 0.5 & 21.00 & 21.78 & 2 nd: & DHIIH & $\mathrm{nI}$ & 11 \\
\hline HS $0040+0952$ & 64.5 & -17.44 & 0.7 & 1.8 & 0.4 & 1.3 & 0.4 & 20.0: & 20.5: & 4 nd:h: & DHIIH & $\mathrm{nE}$ & 12 \\
\hline HS $0043+0531$ & 165.1 & -18.59 & 1.8 & 4.0 & 1.1 & 1.4 & 1.1 & 20.18 & 20.40 & $1 \mathrm{~d}$ & HIIH & $\mathrm{nI}$ & 12 \\
\hline HS $0051+0927$ & 82.8 & -17.18 & 0.7 & 1.7 & 0.4 & 1.0 & 0.4 & 19.26 & 19.23 & $3 \mathrm{c}: \mathrm{d}:$ & DHIIH & $\mathrm{i}: \mathrm{E}$ & 12 \\
\hline HS $0052+2119$ & 176.1 & -19.01 & 1.9 & 4.3 & 1.3 & 1.2 & 1.1 & 19.53 & 19.83 & $3 \mathrm{c}: \mathrm{d}:$ & DANS/HIIH & $\mathrm{nI}$ & 12 \\
\hline HS $0058+1847$ & 152.7 & -18.76 & 1.3 & 3.3 & 0.7 & & & & & $4 \mathrm{st}$ & DANS/HIIH & $\mathrm{nE}$ & 12 \\
\hline HS $0110+2149$ & 227.5 & -22.12 & 7.6 & 20.0 & 1.0 & 7.4 & 8.2 & 19.5: & 19.7: & $2 \mathrm{~b}: \mathrm{d}: \mathrm{h}$ & SBN(Sy?) & $\mathrm{nI}$ & 12 \\
\hline HS $0111+2115$ & 129.5 & -19.71 & 2.6 & 5.6 & & & & & & $3 \mathrm{cnv}$ & HIIH/MI & iI & 21 \\
\hline HS $0113+1750$ & 252.5 & -19.06 & 1.9 & 4.4 & 0.6 & & & & & $4 \mathrm{st}$ & DANS & $\mathrm{nE} / \mathrm{i} 0$ & 12 \\
\hline HS $0123+1624$ & 118.0 & -18.75 & 1.7 & 4.1 & 1.2: & 1.7 & 0.9 & 19.66 & 20.39 & $1 \mathrm{~d}$ & HIIH & iI & 11 \\
\hline HS $0133+1341$ & 97.4 & -18.42 & 1.0 & 2.9 & 0.6 & 2.0 & 0.9 & 20.61 & 20.74 & 2 nd: & DANS & $\mathrm{nE}$ & 12 \\
\hline HS $0143+2400$ & 140.5 & -18.15 & 1.1 & 2.9 & 0.6 : & 1.3 & 0.9 & 20.48 & 21.04 & $2 \mathrm{nd}$ & DHIIH & iI & 11 \\
\hline HS $0148+1700$ & 260.6 & -21.00 & 2.9 & 7.9 & & 1.2 & 1.5 & 20.3: & 20.7: & 2 bn:d: & SBN/GI & iI,C: & 31 \\
\hline HS $0148+2123$ & 68.0 & -18.12 & 0.9 & 2.5 & 0.7 & 2.3: & $0.8:$ & 20.5: & 20.7: & 2 nd: & DHIIH/HIIH & $\mathrm{iE}: \mathrm{C}$ & 31 \\
\hline
\end{tabular}

